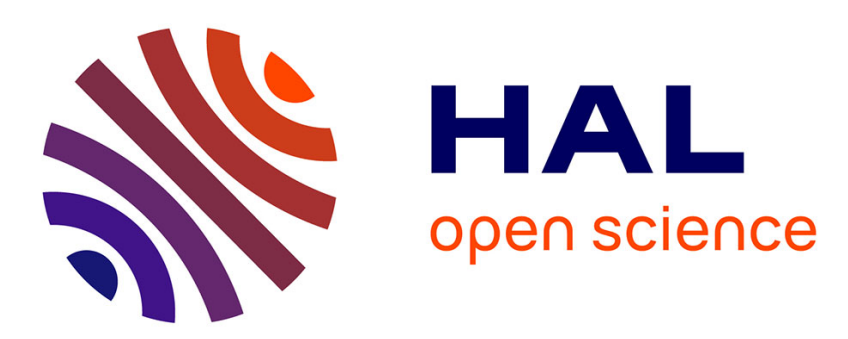

\title{
Risk aversion, the value of information and traffic equilibrium
}

\author{
André de Palma, Robin Lindsey, Nathalie Picard
}

\section{To cite this version:}

André de Palma, Robin Lindsey, Nathalie Picard. Risk aversion, the value of information and traffic equilibrium. 2008. hal-00349492

\section{HAL Id: hal-00349492 \\ https://hal.science/hal-00349492}

Preprint submitted on 31 Dec 2008

HAL is a multi-disciplinary open access archive for the deposit and dissemination of scientific research documents, whether they are published or not. The documents may come from teaching and research institutions in France or abroad, or from public or private research centers.
L'archive ouverte pluridisciplinaire HAL, est destinée au dépôt et à la diffusion de documents scientifiques de niveau recherche, publiés ou non, émanant des établissements d'enseignement et de recherche français ou étrangers, des laboratoires publics ou privés. 


\title{
ECOLE POLYTECHNIQUE
}

\section{RISK AVERSION, THE VALUE OF INFORMATION AND} TRAFFIC EQUILIBRIUM

\author{
André de Palma \\ Robin Lindsey \\ Nathalie Picard
}

December 2008

Cahier $n^{\circ} \quad 2008-22$

\section{DEPARTEMENT D'ECONOMIE}

Route de Saclay

91128 PALAISEAU CEDEX

(33) 169333033

http://www.enseignement.polytechnique.fr/economie/

mailto:chantal.poujouly@polytechnique.edu 


\title{
Risk aversion, the value of information and traffic equilibrium*
}

\author{
André de Palma ${ }^{\dagger} \quad$ Robin Lindsey ${ }^{\ddagger} \quad$ Nathalie Picard ${ }^{\S}$
}

December 13, 2008

\begin{abstract}
Information about traffic conditions has traditionally been conveyed to drivers by radio and variable message signs, and more recently via the Internet and Advanced Traveler Information Systems. This has spurred research on how travelers respond to information, how much they are willing to pay for it and how much they are likely to benefit from it collectively. In this paper we analyze the decisions of drivers whether to acquire information and which route to take on a simple congested road network. Drivers vary in their degree of risk aversion with respect to travel time. Four information regimes are considered: No information, Free information which is publicly available at no cost, Costly information which is publicly available for a fee, and Private information which is available free to a single individual. Private information is shown to be individually more valuable than either Free or Costly information, while the benefits from Free and Costly information cannot be ranked in general. Free or Costly information can decrease the expected utility of drivers who are very risk-averse, and with sufficient risk aversion in the population the aggregate compensating variation for information can be negative.
\end{abstract}

\section{Introduction}

For decades information about driving conditions and traffic delays has been provided to drivers by radio, TV and variable message signs. It has also long been recognized that the relation between individual and aggregate drivers' behaviour depends on the specification of information availability (see the seminal paper of Mahmassani et al., 1986). More recently, information has become available via telephone information systems, Advanced Traveler Information Systems (ATIS) and the Internet. The advent of modern communications technology has spurred research on how travelers respond to information, how much they are willing to pay for it and how much they are likely to benefit from it individually and collectively. Because information affects individual travel decisions, individual travel decisions collectively affect travel conditions, and travel conditions determine what information should be conveyed, a complex and recursive set of interdependencies exists.

Due to these complexities much of the research on ATIS has considered simple road networks and focused on just one or two dimensions of travel behaviour. Furthermore, it is usually assumed that travelers seek to minimize their expected travel costs. In a context where route choice is a decision variable this implies that travelers choose

*We would like to thank the editor Hani Mahmassani, associate editor, two anonymous referees, Simon Anderson and Frédéric Koessler for vey helpful comments and suggestions. Financial support from ANR (Agence Nationale de la Recherche), "RiskAttitude" Grant ANR-05-BLAN-0345-01, the Institut Universitaire de France and the Social Sciences and Humanities Research Council of Canada is gratefully acknowledged.

${ }^{\dagger}$ Ecole Normale Supérieure, Cachan, and Ecole Polytechnique, CECO, France.

$\ddagger$ University of Alberta, Canada.

$\S$ THEMA, University of Cergy-Pontoise, and INED, France. 
a route with the lowest expected travel time. But it is unrealistic to assume that expected travel time is the only criterion for route choice (Jackson and Jucker, 1981; Abdel-Aty et al., 1997), and a number of recent studies (e.g., Bates et al, 2001; Lam and Small, 2001; Brownstone and Small, 2005; Small et al., 2005; de Palma and Picard, 2005) provide convincing empirical evidence that travelers dislike not only travel time but also uncertainty about travel time. de Palma and Picard (2006) incorporate travel time uncertainty into the analysis of information systems with endogenous route choice by assuming that travelers are risk averse and seek to maximize their expected utility where utility is a decreasing and concave function of travel time. However, these authors do not analyze the decisions of drivers whether to acquire information or the welfare impacts of information as a function of its cost.

This paper builds on de Palma and Picard (2006) by assuming that information is costly and considering the decisions of drivers whether to purchase it. The paper therefore spans the literature on demand for information with endogenous route choice with risk-neutral drivers (e.g., Yang, 1998; Lo et al., 1999; Lo and Szeto, 2002) and the literature that adopts a utility-theoretic approach with risk-averse drivers but does not analyze demand for information (e.g., Mirchandani and Soroush, 1987; Kobayashi, 1994; Tatineni et al., 1997; Yin and Ieda, 2001a,b; Chen et al., 2002; Yin et al., 2004). In our model, individuals have a choice between a "safe" route, and a "risky" route with a travel time that fluctuates from day to day according to a known probability and according to known road performance functions. Individuals are risk averse and weigh average travel time and variability of travel time when choosing a route. Individuals differ in their degrees of risk aversion so that they trade off expected travel time and variability of travel time at different rates. By combining costly information and risk aversion, the paper takes an important step towards a realistic analysis of the developing market for driver information systems that may ultimately provide significant benefits to drivers in terms of quicker and more predictable trips. Heterogeneity in risk aversion effectively plays an analogous role to heterogeneity in value of travel time which is an important determinant of travel decisions such as mode choice and whether to use toll lanes. In the model, risk aversion governs whether individuals prefer the "safe" route or the "risky" route in the absence of information, and how much they are willing to pay for information about conditions on the "risky" route. By modeling risk aversion the paper accounts not only for the benefits from ATIS in reducing average travel times but also the benefits (or costs) to drivers from changes in the variability in travel times.

Four information regimes are considered. In the No information regime drivers do not have day-specific information and base their route-choice decisions on the unconditional probability distribution of states which they are assumed to know. The second regime is one of Free information in which all drivers receive free and accurate information about travel conditions each day. In the third regime of Costly information drivers can purchase the information. The analysis focuses on how the purchase decision depends on the fee and the individual degree of risk aversion, as well as on how the route-choice decisions of informed drivers affect the expected utilities of drivers who do not purchase information. The final information regime is one of Private information in which information is available free to a single individual.

Three results stand out. One is that an individual driver always benefits more from Private information than from Free information or Costly information. The second — and less obvious — finding is that both Free information and Costly information leave sufficiently risk-averse drivers worse off even though individually they may be willing to pay for the information. Third, if there are enough highly risk-averse drivers in the population the aggregate compensating variation for information may be negative. All three results derive from the fact that, while information helps drivers to make better decisions individually (and never worse decisions), their collective response to information can exacerbate the distribution and variability of congestion delay on the network, and may even leave drivers collectively worse off.

In summary the analysis is based on a stochastic network deterministic user equilibrium (SN-DUE) model in 
which road network capacity is stochastic and users do not have idiosyncratic perception errors. Route-choice behaviour is based on expected utility theory. The model is static in the sense that users know the probability distribution of states conditional on the information regime so that learning does not take place. Finally, the ATIS provides perfect rather than imperfect information. Although this framework is restrictive it permits the derivation of analytical results that have an intuitive appeal and should help to promote understanding of more complicated simulation, experimental and empirical studies of ATIS.

The paper is organized as follows. Section 2 lays out the model and explains the measurement of welfare change using compensating variation. As a benchmark, Section 3 analyzes the equilibria and welfare properties of the information regimes when drivers are risk-neutral. Section 4 derives equilibria for the No information and Free information regimes when drivers are risk averse, and analyzes the positive and normative impacts of Free information. Section 5 - which constitutes the heart of the paper - conducts a parallel analysis for Costly information. Section 6 considers Private information and compares its benefits with the benefits of Free information and Costly information. Section 7 presents a numerical example that illustrates the theoretical results and conveys a sense of the magnitude of the welfare impacts. Section 8 concludes.

\section{The model}

This section presents the model which is based on de Palma and Picard (2006). It defines the information regimes and explains the measurement of welfare change. Notation is modified and simplified where feasible to streamline extensions in later sections.

\section{$1.1 \quad$ Assumptions}

A continuum of drivers with mass $N$ travels from a common origin to a common destination. $N$ is fixed and exogenous. Each driver has to choose between a safe route, $S$, with a deterministic and known travel time, $t_{S}$, and a risky route, $R$, with a stochastic travel time, $T_{R}$, which depends on the state. There are two states: a good state denoted "-" with a travel time $t_{R}^{-}$, and a bad state denoted "+" with a larger travel time $t_{R}^{+}>t_{R}^{-}$. For brevity the two states will hereafter be called Good days and Bad days. The probability of a Bad day is $p \in(0,1)$ which is assumed fixed and exogenous. Thus:

$$
\mathbb{P}\left(T_{R}=t_{R}^{+}\right)=p \text {, and } \mathbb{P}\left(T_{R}=t_{R}^{-}\right)=1-p .
$$

There is congestion on route $S$, as well as on route $R$ on $B a d$ days, in the sense that travel time on route $j$ increases with the number, $N_{j}$, of users who choose route $j, j=R, S$. However, there is no congestion on route $R$ on Good days: $t_{R}^{-}$is constant. To formalize:

Assumption 1 Travel time $t_{S}\left(N_{S}\right)$ is continuous and strictly increasing in $N_{S}$. On Good days travel time on route $R$ is a constant $t_{R}^{-}$. On Bad days, travel time on route $R$ is a continuous and strictly increasing function $t_{R}^{+}\left(N_{R}\right)$ of $N_{R}$.

Therefore, $t_{R}^{+}(0)$ represents free-flow travel time on route $R$ on bad days. Three further assumptions are made about travel times. First, on Good days route $R$ is faster than $S$ even with $N_{S}=0$. Second, on $B a d$ days travel time on $R$ is always longer than on Good days even with $N_{R}=0$. Finally, if all drivers choose one route on a $B a d$ day then it is slower than the other (unused) route. Therefore: 
Assumption $2 t_{R}^{-}<t_{S}(0)<t_{R}^{+}(N)$ and $t_{R}^{-}<t_{R}^{+}(0)<t_{S}(N)$.

No assumption is made about the relative magnitudes of $t_{S}(0)$ and $t_{R}^{+}(0)$ or $t_{R}^{+}(N)$ and $t_{S}(N)$.

Assumptions 1 and 2 are illustrated in Figure 1. The number of drivers taking route $R, N_{R}$, is measured in the usual way to the right from the left-hand vertical axis, while the number taking route $S$, $N_{S}$, is measured to the left from the right-hand axis. Travel times are measured on the vertical axes. Functions $t_{R}^{+}\left(N_{R}\right)$ and $t_{S}\left(N_{S}\right)$ intersect where $N_{R}>0$ and $N_{S}>0$, and lie wholly above the horizontal line at $t_{R}^{-}$.

Figure 1 about here

Drivers' preferences are specified in:

Assumption 3 Drivers' preferences are represented by a differentiable utility function $U(t ; \theta)$ where $\theta \geq 0$ is the risk aversion parameter. For $\theta=0, U(t ; 0)=-(a+t)$, where $a \geq 0$. For $\theta>0$ and $t>0, U(t ; \theta)$ is strictly decreasing and strictly concave in $t$. Writing $U(t ; \theta)$ as $U(u ; \theta)$ where $u=U(t ; \theta)$, for $\theta>0:(a) \partial U(t ; \theta) / \partial \theta$ is a strictly concave function of $u$ and $(b) \operatorname{Lim}_{\theta \rightarrow \infty} U\left(t_{2} ; \theta\right) / U\left(t_{1} ; \theta\right)=\infty$, for $t_{2}>t_{1}>0$.

Property (a) in the last sentence of Assumption 3 is used to prove Lemma 1 below. Property (b) is a technical condition. It formalizes the idea that additional travel time is disproportionately costly for an "infinitely" riskaverse individual. It is straightforward to check (see Appendix 1) that Assumption 3 is satisfied for Hyperbolic Absolute Risk Aversion (HARA) preferences which include Constant Relative Risk Aversion (CRRA) preferences and Constant Absolute Risk Aversion (CARA) preferences as special cases. For HARA the utility function is $U(t ; \theta)=-\frac{(a+t)^{1+\theta}}{1+\theta}, a \geq 0$, for CRRA it is $U(t ; \theta)=-\frac{t^{1+\theta}}{1+\theta}$, and for CARA it is $U(t ; \theta)=\frac{1-\exp (t \theta)}{\theta}$. CARA preferences will be adopted in the numerical example of Section 6 .

Expected utility on a route is $\mathbb{E} U(T ; \theta)=p U\left(T^{+} ; \theta\right)+(1-p) U\left(T^{-} ; \theta\right)$, where $T^{+}$denotes travel time on the route on Bad days, and $T^{-}$denotes travel time on Good days. The distribution of $\theta$ in the population is described in:

Assumption 4 The risk aversion parameter $\theta$ has a continuous distribution over an interval $\mathcal{I}$, with either $\mathcal{I}=$ $\mathbb{R}^{+}$or $\mathcal{I}=\left[0 ; \theta^{M}\right] \subset \mathbb{R}^{+}$for $\theta^{M} \in[0, \infty)$. The distribution is characterized by the strictly increasing CDF $F(\theta) \in[0 ; 1], \forall \theta \in \mathcal{I}$, and by the density $f(\theta)>0, \forall \theta$ in the interior of $\mathcal{I}$. If the distribution is bounded then $\mathbb{E} U\left(T_{R}(N) ; \theta^{M}\right)<U\left(t_{S}(0) ; \theta^{M}\right)$.

The last sentence of Assumption 4 implies that either expected travel time is larger on $R$ when all drivers select $R$, or $\theta^{M}$ is sufficiently large. This assumption is necessary for an interior equilibrium to exist. Otherwise, all the users would select $R$ when the state is unknown. No similar assumption is necessary when $\theta$ is distributed over $\mathbb{R}^{+}$ since the assumption $t_{R}^{+}(N)>t_{S}(0)$ guarantees that a sufficiently risk-averse driver prefers route $S$ to route $R$.

Before turning to the information regimes some justification for the assumptions is in order. As noted in the introduction, restrictive assumptions are required for the model to be analytically tractable and the assumptions chosen allow us to concentrate on the effects of drivers' aversion to travel time variability. The assumption that the number of users, $\mathrm{N}$, is fixed means that travel demand is deterministic and price inelastic. This is a common assumption in the literature on ATIS and it is reasonable in settings where travelers have few alternatives to driving. Some other studies (e.g., Emmerink et al., 1996; Zhang and Verhoef, 2006) adopt models with elastic travel demand while treating route and other choices as fixed. 
Stochasticity is manifest in the model only on route $R$. On Good days travel time on route $R$ is constant, and on Bad days there is congestion and travel time is higher. Route $R$ can be thought of as a multilane freeway that, under ideal conditions (Good days), has adequate capacity to handle all traffic ( $N$ users) at free-flow speeds. But when conditions are impaired (Bad days), capacity is reduced and congestion develops. (According to Assumption 2, free-flow travel time is also higher than on Good days.) Non-ideal conditions can arise for various reasons: bad weather that makes the surface slippery, reduces visibility or requires snow plowing; natural disasters such as rock slides; accidents or disabled vehicles that block one or more lanes; unscheduled road maintenance and so on. The probability of Bad days is treated as exogenous. This is reasonable for acts of nature, but problematic for accidents and other anthropogenic causes. Nevertheless, most existing ATIS studies treat state probabilities as exogenous. Moreover, empirical studies of the relationship between the frequency of accidents and traffic volumes have produced mixed results. The additional assumption that drivers know the probabilities allows us to focus on risk rather than uncertainty. If the probabilities were unknown, learning would take place, and this would make the problem far more complex (see Chancelier et al., 2007).

The assumption that travel time on route $S$ is deterministic also deserves comment. One possibility is that route $S$ is equipped with emergency or breakdown lanes that can accommodate disabled vehicles and vehicles involved in accidents without reducing throughput. The assumption may also be reasonable if route $S$ is located in a corridor that is not susceptible to landslides, fog and other forces that cause Bad days on route $R$.

Assumption 3 stipulates that drivers' preferences are defined in terms of utilities rather than costs and that drivers choose the route with the higher expected utility. Although expected utility theory is now being challenged in the field of transportation (e.g. Avineri and Prashker, 2004, 2005; see also de Palma et al., 2008), it is still the most common paradigm. Note, however, that the model developed here (based on a binary distribution of travel time) trivially extends to the most common deviations to expected utility: probability weighting, prospect theory and cumulative prospect theory. Introducing expected and non expected utility in Random Utility Models and other Discrete Choice Models remains an open and challenging topic (see de Palma et al., 2008). Note that expected utility maximization includes expected travel cost minimization as a special case that is analyzed as a benchmark in Section 3.

\subsection{Information regimes}

Four information regimes will be considered that differ according to drivers' knowledge of the state when they choose a route:

No information $(Z)$ : drivers know the probability $p$, but not the actual state.

Free information $(F)$ : all drivers are informed about the state at no cost.

Costly information $(C)$ : any driver can learn the state at a cost $\pi$.

Private information $(I)$ : a single driver learns the state at no cost.

It is assumed that for each information regime, all drivers know the probability distribution of travel times on the two routes conditional on the information regime and whether they are personally informed. (It is not necessary for drivers to know the distribution of risk attitudes in the population or for them to predict what route each individual driver will choose. All they need to know is aggregate usage on each route in each state.) With Private information the equilibrium is the same as with No information since, with a continuum of drivers, the route choice of one driver does not affect traffic conditions.

In the case of Costly information it is assumed that the price of information is equivalent to an increase in travel time, with the value of time (VOT) normalized to unity and hence independent of $\theta$. Expected utility can 
then be written $\mathbb{E} U(T+\pi ; \theta)$. This specification incorporates two underlying assumptions. One is that VOT is independent of the duration of a trip and the same for uncongested and congested travel time. The second is that individuals have the same risk attitudes towards travel time and monetary cost. There is little empirical evidence on which to judge the validity of this second assumption. Aversion to travel time uncertainty is now well documented in the literature. In general people also dislike uncertainty about payment even when the amount at stake is small. However, a majority of drivers are favorably disposed towards High Occupancy Toll (HOT) lane facilities in the U.S. where tolls vary dynamically in near real-time to maintain free-flow conditions. Users of the toll lanes therefore purchase a (nearly) risk-free travel time in exchange for an uncertain price. This type of opportunity is not available on other tolled facilities or in the model used here, and driver attitudes may differ.

Denote usage of the two routes on Good days by $N_{R}^{-}$and $N_{S}^{-}$, and on $B a d$ days by $N_{R}^{+}$and $N_{S}^{+}$. For all regimes except Free information the division of traffic between routes is characterized by:

Proposition 1 On Bad days, in the No information, Costly information and Private information regimes, traffic $N_{R}^{+}$on $R$ is such that $t_{R}^{+}\left(N_{R}^{+}\right)>t_{S}\left(N_{S}^{+}\right)$.

Proof. Assumptions 1 and 2 imply that route $R$ is preferred by all users to route $S$ on Good days even if everyone takes $R$. If $R$ were also preferred to $S$ on $B a d$ days, then $R$ would be preferred to $S$ whatever the state. But this implies $N_{R}^{+}=N$ and $t_{R}^{+}(N) \leq t_{S}(0)$, which contradicts Assumption 2 .

Depending on their preferences and on $\pi$, in the Costly information regime drivers choose between three strategies:

- Strategy $R$ ( $n_{R}$ drivers): Do not pay for information and choose route $R$ in both states.

- Strategy $S$ ( $n_{S}$ drivers): Do not pay for information and choose route $S$ in both states.

- Strategy I ( $n_{I}$ drivers): Pay for information. Given Assumption 2 and Proposition 1, the best choice when informed is route $R$ on Good days and route $S$ on Bad days.

The following conservation laws apply: $n_{R}+n_{S}+n_{I}=N_{R}^{+}+N_{S}^{+}=N_{R}^{-}+N_{S}^{-}=N$.

Traffic $N_{j}^{q}$ on route $j$ when the state is $q$ is therefore as given in Table 1:

\begin{tabular}{|l|l|l|}
\hline Route $j \backslash$ State $q$ & Bad days $(q="+")$ & Good days $(q="-")$ \\
\hline$j=R$ & $N_{R}^{+}=n_{R}$ & $N_{R}^{-}=n_{R}+n_{I}=N-n_{S}$ \\
\hline$j=S$ & $N_{S}^{+}=n_{S}+n_{I}=N-n_{R}$ & $N_{S}^{-}=n_{S}$ \\
\hline
\end{tabular}

Table 1: Route split in Costly information regime

The strategies, the numbers of drivers choosing each strategy, their route choices and expected utilities in the four information regimes are summarized in Table 2. Note that Strategies $R$ and $S$ are equivalent to a route choice in both states. With Strategy $I$, drivers select route $R$ on Good days and route $S$ on Bad days. (With Free information all drivers are informed at no cost. Since, however, $n_{R}^{F+}$ drivers take route $R$ on $B a d$ days they can be thought of as choosing Strategy $R$ and ignoring the state.) 


\begin{tabular}{|c|c|c|c|l|}
\hline Strategy & $\begin{array}{c}\text { Numbers } \\
\text { of drivers }\end{array}$ & $\begin{array}{c}\text { Bad } \\
\text { days }\end{array}$ & $\begin{array}{c}\text { Good } \\
\text { days }\end{array}$ & Expected utility \\
\hline \multicolumn{5}{|c|}{ No information } \\
\hline$R$ & $n_{R}^{Z}$ & $R$ & $R$ & $p U\left(t_{R}^{+}\left(n_{R}^{Z}\right) ; \theta\right)+(1-p) U\left(t_{R}^{-} ; \theta\right)$ \\
\hline$S$ & $n_{S}^{Z}$ & $S$ & $S$ & $U\left(t_{S}\left(n_{S}^{Z}\right) ; \theta\right)$ \\
\hline \multicolumn{6}{|c|}{ Free information } \\
\hline$R$ & $n_{R}^{F+}=N_{R}^{+}$ & $R$ & $R$ & $p U\left(t_{R}^{+}\left(n_{R}^{F+}\right) ; \theta\right)+(1-p) U\left(t_{R}^{-} ; \theta\right)$ \\
\hline$I$ & $n_{I}^{F+}=N_{S}^{+}$ & $S$ & $R$ & $p U\left(t_{S}\left(n_{I}^{F+}\right) ; \theta\right)+(1-p) U\left(t_{R}^{-} ; \theta\right)$ \\
\hline \multicolumn{5}{|c|}{ Costly information } \\
\hline$R$ & $n_{R}^{C}$ & $R$ & $R$ & $p U\left(t_{R}^{+}\left(n_{R}^{C}\right) ; \theta\right)+(1-p) U\left(t_{R}^{-} ; \theta\right)$ \\
\hline$S$ & $n_{S}^{C}$ & $S$ & $S$ & $p U\left(t_{S}\left(n_{S}^{C}+n_{I}^{C}\right) ; \theta\right)+(1-p) U\left(t_{S}\left(n_{S}^{C}\right) ; \theta\right)$ \\
\hline$I$ & $n_{I}^{C}$ & $S$ & $R$ & $p U\left(t_{S}\left(n_{S}^{C}+n_{I}^{C}\right)+\pi ; \theta\right)+(1-p) U\left(t_{R}^{-}+\pi ; \theta\right)$ \\
\hline \multicolumn{5}{|c|}{$\operatorname{Private~information}$} \\
\hline$R$ & $n_{R}^{Z}$ & $R$ & $R$ & $p U\left(t_{R}^{+}\left(n_{R}^{Z}\right) ; \theta\right)+(1-p) U\left(t_{R}^{-} ; \theta\right)$ \\
\hline$S$ & $n_{S}^{Z}$ & $S$ & $S$ & $U\left(t_{S}\left(n_{S}^{Z}\right) ; \theta\right)$ \\
\hline$I$ & Single driver & $S$ & $R$ & $p U\left(t_{S}\left(n_{S}^{Z}\right) ; \theta\right)+(1-p) U\left(t_{R}^{-} ; \theta\right)$ \\
\hline
\end{tabular}

Table 2: Strategies, numbers of drivers, route choices and expected utilities

\subsection{Measuring welfare change}

Willingness to pay is measured in economics using compensating variation and equivalent variation (see Varian 1992, Chapter 10). The compensating variation (CV) is defined to be the amount an individual is willing to pay for a change to take place, whereas equivalent variation (EV) is defined to be the amount an individual requires in order to be as well off as if a change takes place. Compensating variation will be adopted here since it is assumed when assessing the Free, Costly and Private information regimes that information is actually provided so that the change does take place. CV will be measured in time units in the same way that the cost of information is measured; thus it corresponds to the additional certain travel time that a driver is willing to incur for information. Since the value of time (VOT) is assumed to be constant, if desired CV can be translated into monetary units by multiplying the CV by the VOT.

Definition 1 The individual compensating variation for information regime $r, C V^{r}(\theta), r \in\{F, C, I\}$, corresponds to the additional time an individual with utility $U(. ; \theta)$ is willing to incur for a shift to regime $r$ from the No information regime. $C V^{r}(\theta)$ is defined implicitly by the condition $\mathbb{E} U^{r}\left(T+C V^{r}(\theta) ; \theta\right)=\mathbb{E} U^{Z}(T ; \theta)$.

(Note that the corresponding definition for $\mathrm{EV}$ is $\mathbb{E} U^{r}(T ; \theta)=\mathbb{E} U^{Z}\left(T-E V^{r}(\theta) ; \theta\right)$ which does not yield the same value as CV unless $U(. ; \theta)$ is linear; i.e. the individual is risk-neutral.) The CV for information depends, $a$ priori, on who receives it, and the CVs for Free, Costly and Private information all differ in general. Naturally, proprietary information is never unfavorable to an individual. But when other individuals also have access to information, they may respond to it in ways that exacerbate the distribution of congestion on the network.

\section{Risk-neutral drivers}

To develop a preliminary understanding of the model, as well as to provide a benchmark against which to assess the implications of risk aversion, it is instructive to identify and characterize equilibria for the four information 
regimes when drivers are risk neutral. (Risk neutrality is a limiting case of the model with a compact distribution of risk aversion with parameter $\theta^{M}$ in Assumption 4 set to zero.)

\subsection{Free information}

In the Free information regime all drivers choose route $R$ on Good days and incur a travel cost $C^{F-}=t_{R}^{-}$. The equilibrium corresponds to point $F_{R}^{-}$in Figure 2 with coordinates $\left(n_{R}=N, t_{R}^{-}\right)$, where the subscript $R$ on $F_{R}^{-}$indicates that the outcome is realized on Route $R$. On Bad days, drivers divide themselves between routes according to Wardrop's principle so that travel costs are equalized: $C^{F+}=t_{R}^{+}\left(n_{R}^{F+}\right)=t_{S}\left(n_{S}^{F+}\right)=t_{S}\left(N-n_{R}^{F+}\right)$. The corresponding point in Figure 2 is $F_{R, S}^{+}$where the subscript $R, S$ indicates that the outcome is realized on both routes. Expected travel costs are $\mathbb{E} C^{F}=(1-p) C^{F-}+p C^{F+}=(1-p) t_{R}^{-}+p t_{R}^{+}\left(n_{R}^{F+}\right)$.

Figure 2 about here

\subsection{No information and Private information}

If drivers do not know the state then they do not know actual travel costs on each route. However, if drivers are risk neutral, they are only concerned with expected travel cost, and they prefer the route with lower expected travel cost. Following Emmerink (1998) it is assumed that Wardrop's principle applies in terms of expected travel costs; thus, drivers allocate themselves between routes so that expected travel costs are equal: $\mathbb{E} C^{Z}=(1-p) t_{R}^{-}+p t_{R}^{+}\left(n_{R}^{Z}\right)=$ $t_{S}\left(N-n_{R}^{Z}\right)$. (To obtain $n_{S}^{Z}=N-n_{R}^{Z}>0$, the probability of Bad days must satisfy $p>\frac{t_{S}(0)-t_{R}^{-}}{t_{R}^{+}(N)-t_{R}^{-}}$.) Point $Z_{S}^{\bullet}$ identifies the equilibrium for users of Route $S$. This outcome is certain because travel costs are the same in both states. Point $Z_{R}^{-}$identifies the outcome for users of Route $R$ on Good days, and $Z_{R}^{+}$the outcome on Bad days. The comparative statics properties of the equilibrium are derived in Appendix 2 and presented in Table 3.

\begin{tabular}{|l|c|c|}
\hline & $p$ & $N$ \\
\hline$n_{R}$ & $<0$ & $\in(0,1)$ \\
\hline$n_{S}$ & $>0$ & $\in(0,1)$ \\
\hline$E \cdot C^{Z}$ & $>0$ & $>0$ \\
\hline
\end{tabular}

Table 3: Comparative statics properties of No information equilibrium with risk neutral drivers

As expected, an increase in the probability of $B a d$ days reduces usage of route $R$, increases usage of route $S$, and increases expected travel costs. If the number of drivers increases, usage of both routes increases as do expected travel costs.

With risk-neutral drivers the CV for Free information is simply the difference in expected costs between the No information and Free information regimes: $C V^{F}=\mathbb{E} C^{Z}-\mathbb{E} C^{F}=p\left(t_{R}^{+}\left(n_{R}^{Z}\right)-t_{R}^{+}\left(n_{R}^{F+}\right)\right)>0 . C V^{F}$ is positive because on $B a d$ days fewer drivers take route $R$; i.e. $n_{R}^{F+}<n_{R}^{Z}$. The welfare gain can be decomposed into three parts. First, on Good days information benefits drivers who would otherwise take route $S$. This benefit is identified by the speckled area labeled $B^{-}$in the lower right of Figure 2. Second, on Bad days information benefits drivers who take route $R$ as indicated by the speckled area $B^{+}$in the upper left. Finally, information imposes a loss on drivers who take route $S$ as shown by the shaded area $L^{+}$to the upper right. (Area $B^{-}$corresponds to what Zhang and Verhoef (2006) call decision-making benefits. Areas $B^{+}$and $L^{+}$correspond to what they call travel cost benefits (or costs).) The loss $L^{+}$is outweighed by the benefits $B^{-}$and $B^{+}$. To see this geometrically note that area $B^{-}$(weighted by the probability of Good days) equals the area of the rectangle defined by points $Z_{S}^{\bullet}$, 
$Z_{R}^{+}, t_{R}^{Z+}$ and $t_{S}^{Z}$ (weighted by the probability of $B a d$ days) which exceeds area $L^{+}$. Area $B^{-}$thus outweighs area $L^{+}$, and area $B^{+}$adds to the net benefit.

Free information reduces expected travel time, as well as variability of travel time on route $R$. But it also raises travel time on $B a d$ days on route $S$ (since $t_{S}\left(n_{S}^{F+}\right)>t_{S}\left(n_{S}^{Z}\right)$ ) and results in travel time uncertainty for all drivers. This uncertainty is of no consequence per se for risk-neutral drivers, but it does matter with risk-averse drivers as will be shown in Section 4.

Before turning to Costly information consider the Private information regime. If a single driver is informed he can take route $R$ on Good days, and route $S$ on Bad days. The CV for Private information is $C V^{I}=\mathbb{E} C^{Z}-\mathbb{E} C^{I}$ $=p\left(t_{R}^{+}\left(n_{R}^{Z}\right)-t_{S}\left(n_{S}^{Z}\right)\right)>0 . C V^{I}$ exceeds the CV for Free information since $t_{S}\left(n_{S}^{Z}\right)<t_{R}^{+}\left(n_{R}^{F+}\right)=t_{S}\left(n_{S}^{F+}\right)$. Information is always valuable when it is private because the benefits of shifting to route $S$ on $B a d$ days are not dissipated by similar adjustments of other drivers. As will be seen, the advantage of Private information over Free information holds - and with greater force - when drivers are risk-averse.

\subsection{Costly information}

Equilibrium for the Costly information regime with risk-neutral drivers is derived in Appendix 2. In order for some drivers to be willing to purchase information (Strategy $I$ ) the cost cannot be too high; otherwise the equilibrium is the same as with No information. Regardless of the cost of information some drivers choose not to purchase it and stick to route $R$ (Strategy $R$ ). If the cost of information exceeds a lower bound then some drivers choose Strategy $S$; otherwise all drivers adopt either Strategy $R$ or Strategy $I$. The comparative statics properties of equilibrium are presented in Table 4. Predictably, as the cost of information increases, $n_{I}^{C}$ decreases and $n_{R}^{C}$ and $n_{S}^{C}$ increase. As the probability of Bad days increases, $n_{S}^{C}$ rises and $n_{R}^{C}$ falls. As shown in Appendix $2, n_{I}^{C}$ increases if $p$ is small, and decreases if $p$ is large. This is because travel time uncertainty is greatest for intermediate values of $p$. Perhaps surprisingly, $n_{S}$ is independent of $N$. To see why, note that the choice between strategies $I$ and $S$ depends on the cost of information relative to the travel time saved on Good days from taking route $R$. Since both the cost of information and travel time on $R$ on Good days are constants, travel time on route $S$ must also be constant and hence usage of $S$ must be independent of $N$. This result does not obtain with risk-averse drivers as shown below.

\begin{tabular}{|l|c|c|c|}
\hline & $\pi$ & $p$ & $N$ \\
\hline$n_{R}$ & $>0$ & $<0$ & $\in(0,1)$ \\
\hline$n_{S}$ & $>0$ if $n_{S}>0$ & $>0$ if $n_{S}>0$ & 0 \\
\hline$n_{I}$ & $<0$ & 0 if $n_{S}=0$ & $\begin{array}{l}>0 \text { if } n_{S}>0 \text { and } p \text { small } \\
<0 \text { if } n_{S}>0 \text { and } p \text { large } \\
>0 \text { if } n_{S}=0\end{array}$ \\
\hline $\mathbb{E} C^{C}$ & $>0$ & $>0$ & $\in(0,1)$ \\
\hline
\end{tabular}

Table 4: Comparative statics properties of Costly information equilibrium with risk-neutral drivers

Equilibrium with Costly information is depicted in Figure 3 for the case with $n_{S}^{C}>0$. On Good days, Groups $R$ and $I$ take Route $R$ and realize an outcome at point $C_{R, I}^{-}$. Group $S$ incurs a higher cost at point $C_{S}^{-}$. On Bad days, Group $R$ ends up at point $C_{R}^{+}$, while Groups $S$ and $I$ incur a lower cost at point $C_{S, I}^{+}$. Group $I$ enjoys the best of both worlds, but pays a fee $\pi$ for the privilege.

Figure 3 about here 
Since expected travel costs are the same for all three groups, the CV for Costly information can be computed for any one of them. For Group $R$, expected costs are $\mathbb{E} C_{R}^{C}=(1-p) t_{R}^{-}+p t_{R}^{+}\left(n_{R}^{C}\right)$, and hence $C V^{C}=\mathbb{E} C^{Z}-\mathbb{E} C_{R}^{C}$ $=p\left(t_{R}^{+}\left(n_{R}^{Z}\right)-t_{R}^{+}\left(n_{R}^{C}\right)\right)>0 . C V^{C}$ is positive, but it is smaller than for Free information since $n_{R}^{C}>n_{R}^{F+}$. This is apparent in Figure 3 from the fact that area $B^{+}$is thinner than in Figure 2. For Group $S$ travel costs are higher on Good days than with Free information $\left(t_{S}^{C-}>t_{R}^{-}\right)$, but lower on Bad days $\left(t_{S}^{C+}<t_{S}^{F+}\right)$. Consequently, Group $S$ suffers less variability in costs - which is advantageous if they are risk averse as is the case in the general model.

\section{No information and Free information with risk-averse drivers}

This section establishes some properties of the No information and Free information regimes when drivers are risk averse and examines the CV for Free information. Some of the results generalize results in de Palma and Picard (2006), and other results are new. de Palma and Picard (2006) derive most of their results for CRRA and CARA preferences. The results here are derived under Assumption 3, which includes CRRA and CARA as special cases and permits more compact proofs.

\subsection{Existence and uniqueness of equilibria}

The equilibrium of the Free information regime is described by:

Proposition 2 Consider the Free information regime. Under Assumptions 1 and 2:

(a) On Good days there exists a unique corner equilibrium: $\left(N_{R}^{F-}=N, N_{S}^{F-}=0\right)$.

(b) On Bad days there exists a unique interior equilibrium: $\left(N_{R}^{F+} \in(0, N), N_{S}^{F+}=N-N_{R}^{F+}\right)$, with $t^{+}\left(N_{R}^{F+}\right)=$ $t_{S}\left(N-N_{R}^{F+}\right)$.

Proof. See Appendix 3.

On Good days all drivers prefer route $R$. This follows from Assumption 1 that $t_{R}^{-}$is constant and represents the lowest possible travel time. On $\mathrm{Bad}$ days both routes are used and all drivers are indifferent between them because travel times are equal and there is no uncertainty. The equilibrium is the same as when drivers are risk-neutral (Section 2).

To determine equilibrium for the No information regime it is necessary to identify which individuals take each route. Individual route choice decisions are described by:

Lemma 1 Consider the No information regime. Under Assumptions 1-4, for any $N_{R}$ such that $t_{R}^{+}\left(N_{R}\right)>$ $t_{S}\left(N-N_{R}\right)$ and $p t_{R}^{+}\left(N_{R}\right)+(1-p) t_{R}^{-}<t_{S}\left(N-N_{R}\right)$ :

(a) There exists a unique risk aversion threshold $\tilde{\theta}^{Z}\left(p, N_{R}\right)$ such that $R \succ S \Leftrightarrow \theta<\tilde{\theta}^{Z}\left(p, N_{R}\right)$.

(b) $\tilde{\theta}^{Z}\left(p, N_{R}\right)$ is strictly decreasing in $p$ and $N_{R}$.

Proof. See Appendix 4.

According to Lemma 1(a), whenever expected travel time is lower on $R$ than on $S$ a group of drivers with the least risk aversion prefer to use $R$, and the remainder prefer $S$. The number who use route $R$ is $n_{R}^{Z}=N F\left(\tilde{\theta}^{Z}\left(p, N_{R}\right)\right)$. Lemma 1(b) indicates that $n_{R}^{Z}$ decreases with the probability of Bad days and with usage of $R$.

Equilibrium in the No information regime is described by: 
Proposition 3 Consider the No information regime. Under Assumptions 1-4, there exists a unique equilibrium traffic volume on $R, n_{R}^{Z}(p) \in\left(n_{R}^{F+}, N\right)$, and a unique risk aversion threshold, equal to $\tilde{\theta}^{Z}\left(p, n_{R}^{Z}\right)$, which solve:

$$
\left\{\begin{array}{l}
\text { (a) } F^{-1}\left(n_{R}^{Z} / N\right)=\tilde{\theta}^{Z}\left(p, n_{R}^{Z}\right) \\
\text { (b) } p U\left(t_{R}^{+}\left(n_{R}^{Z}\right) ; \tilde{\theta}^{Z}\left(p, n_{R}^{Z}\right)\right)+(1-p) U\left(t_{R}^{-} ; \tilde{\theta}^{Z}\left(p, n_{R}^{Z}\right)\right)=U\left(t_{S}\left(N-n_{R}^{Z}\right) ; \tilde{\theta}^{Z}\left(p, n_{R}^{Z}\right)\right)
\end{array}\right.
$$

Moreover, $n_{R}^{Z}(p)$ and $\tilde{\theta}^{Z}\left(p, n_{R}^{Z}\right)$ are decreasing in $p$.

Proof. See Appendix 5.

The uniqueness of $\tilde{\theta}^{Z}$ given $n_{R}^{Z}$ follows from Lemma 1. In words, part (a) of Proposition 3 states that drivers with risk aversion less than $\tilde{\theta}^{Z}$ choose route $R$ and the rest choose $S$. Part (b) states that the driver with risk aversion $\tilde{\theta}^{Z}$ is indifferent between the routes.

We now turn to a comparison of the No information and Free information regimes.

Proposition 4 Under Assumptions 1-4:

(a) With Free information, equilibrium usage of route $R$ on Bad days is less than usage with No information: $n_{R}^{F+}<n_{R}^{Z}$

(b) Free information reduces expected travel time for all drivers, and the reduction is larger for the most riskaverse drivers $\left(\theta>\tilde{\theta}^{Z}\left(p, n_{R}^{Z}\right)\right)$ than for the least risk-averse drivers $\left(\theta<\tilde{\theta}^{Z}\left(p, n_{R}^{Z}\right)\right): p t_{R}^{+}\left(n_{R}^{F+}\right)+(1-p) t_{R}^{-}<$ $p t_{R}^{+}\left(n_{R}^{Z}\right)+(1-p) t_{R}^{-}<t_{S}\left(N-n_{R}^{Z}\right)$.

(c) Free information reduces the variability of travel time for the least risk-averse drivers, and increases the variability of travel time for the most risk-averse drivers.

Proof. See Appendix 6.

Part (a) of Proposition 4 is a natural consequence of the fact that route $R$ is impaired on Bad days while route $S$ is unaffected. Part (b) follows from the fact that with No information travel time is higher for the most risk-averse drivers than for the least risk-averse drivers. With respect to Part (c) travel time variability is reduced for the least risk-averse drivers because usage of route $R$ is reduced on $B a d$ days and travel time thus exceeds $t_{R}^{-}$ by a smaller amount. Travel time becomes more variable for other drivers because it does not vary at all with $N_{o}$ information.

The CV for Free information equalizes individual expected utility in the No information and Free information regimes and is therefore driver-specific. Recall that with $N o$ information the $n_{R}^{Z}$ least risk-averse drivers choose route $R$ and incur a random travel time:

$$
T_{R}\left(n_{R}^{Z}\right)=\left\{\begin{array}{c}
t_{R}^{-} \text {on } \text { Good days } \\
t_{R}^{+}\left(n_{R}^{Z}\right) \text { on Bad days }
\end{array},\right.
$$

and the $N-n_{R}^{Z}$ most risk-averse drivers choose route $S$ with deterministic travel time $t_{S}\left(N-n_{R}^{Z}\right)$. In the Free information regime, all users choose $R$ on Good days (with travel time $t_{R}^{-}$) and are indifferent between $R$ and $S$ on $B a d$ days since equilibrium traffic on $R$ on $B a d$ days, $n_{R}^{F+}$, is such that $t_{R}^{+}\left(n_{R}^{F+}\right)=t_{S}\left(N-n_{R}^{F+}\right)$. The individual 
CVs for Free information for groups $R$ and $S, C V_{R}^{F}(\theta)$ and $C V_{S}^{F}(\theta)$, therefore solve:

$$
\begin{aligned}
& R\left(\text { if } \theta<\tilde{\theta}^{Z}\left(p, n_{R}^{Z}\right)\right): \quad p U\left(t_{R}^{+}\left(n_{R}^{F+}\right)+C V_{R}^{F}(\theta) ; \theta\right)+(1-p) U\left(t_{R}^{-}+C V_{R}^{F}(\theta) ; \theta\right)= \\
& S\left(\text { if } \theta>\tilde{\theta}^{Z}\left(p, n_{R}^{Z}\right)\right): \quad p U\left(t_{R}^{+}\left(n_{R}^{F+}\right)+C V_{S}^{F}(\theta) ; \theta\right)+(1-p) U\left(t_{R}^{-}+C V_{S}^{F}(\theta) ; \theta\right)=.
\end{aligned}
$$

The left-hand side of each equation in (2) is expected utility in the Free information regime with CV added to travel time, and the right-hand side is expected utility in the No information regime. The pair of equations in (2) is the counterpart to the equation $C V^{F}=\mathbb{E} C^{Z}-\mathbb{E} C^{F}$ for risk-neutral drivers.

According to Proposition 4, Free information reduces expected travel time for all drivers and the reduction is larger for the most risk-averse drivers who choose route $S$. CV, however, exhibits a different pattern. Under Assumption 3, $C V_{R}^{F}(\theta)$ is an increasing function of $\theta$ and $C V_{S}^{F}(\theta)$ is a decreasing function of $\theta$. CV is therefore highest for drivers with risk aversion $\tilde{\theta}^{Z}\left(p, n_{R}^{Z}\right)$ who are indifferent between strategies $R$ and $S$. Furthermore, $\mathrm{CV}$ is negative for drivers who are sufficiently risk averse. (This follows intuitively from the definition of $C V_{S}^{F}(\theta)$ and the fact that $t_{R}^{+}\left(n_{R}^{F+}\right)=t_{S}\left(n_{S}^{F+}\right)>t_{S}\left(n_{S}^{Z}\right)$ so that drivers who take the safe route fare worse on Bad days when information is provided.) These results are formalized in:

Theorem 1 Under Assumptions 1-4:

(a) The $C V$ for Free information is an increasing function of risk aversion for the least risk-averse drivers who take route $R$ with No information, and a decreasing function of risk aversion for the most risk-averse drivers who take route $S$ with No information.

(b) When the risk aversion parameter is distributed over $\mathbb{R}^{+}$, the $C V$ for Free information is negative for the most risk-averse users.

Proof. Part (a) is proved in Appendix 7. Part (b) is proved in Appendix 8.

The most risk-averse drivers take the safe route in the No information regime. They gain and lose from Free information. They gain on Good days because they can save time by shifting to the risky route. But they lose on $B a d$ days because some of the least risk-averse drivers shift onto the safe route. This increases travel time on the safe route and also increases the variability of travel time that the most risk-averse drivers experience. The benefit on Good days outweighs the loss on Bad days for drivers with intermediate levels of risk aversion. (Recall from Section 3 that this is true of all drivers if drivers are risk-neutral.) But for the very most risk-averse drivers the benefit is outweighed by the loss.

The possibility that providing (better) information about travel conditions on a congested road network can make users worse off is not new. It has been demonstrated in models with endogenous numbers of trips (e.g., Arnott et al., 1996; de Palma and Lindsey, 1998) and with endogenous departure times (e.g., Arnott et al., 1991) as well as endogenous route choice when drivers overreact to information (e.g., Schelling, 1978; Ben-Akiva, de Palma and Kaysi, 1991; Mahmassani and Jayakrishnan, 1991). None of these papers features risk aversion. Emmerink et al. (1998) use a model that does incorporate risk aversion by including in the generalized cost function a term proportional to the standard deviation of travel time. They show that in regimes equivalent to the Free information and Costly information regimes, information makes all drivers better off. However, if an exogenous fraction of drivers is informed the uninformed can be worse off if the cost of travel time variation is sufficiently large. In their model this happens because elimination of uncertainty encourages informed drivers to travel more. 
The basic mechanism whereby drivers can be worse off is similar to the mechanism here although details differ (in their model and example, individuals are homogeneous with respect to risk aversion, the proportion of users who are informed is exogenous, demand and travel cost functions are linear, and the number of trips is endogenous rather than route choice).

In the model here free information is individually welfare-reducing when it induces changes in driver behaviour that exacerbate congestion in a particular way. The expected private benefit that an individual derives from adjusting his or her route choice on the basis of daily travel conditions can be outweighed by the effects of adjustments by other drivers. As the next section demonstrates, this is also possible if drivers have to pay for information.

\section{Costly information with risk-averse drivers}

The Costly information regime is more complicated than the No information regime since drivers can choose between three strategies $(R, S$ and $I)$ rather than just two $(R$ and $S)$. Similar to the approach taken for the $N o$ information regime, equilibrium will be derived in two steps. In the first step, an individual driver's strategy choice for information acquisition and route selection is derived while holding fixed the numbers of drivers who adopt each strategy (i.e. $n_{R}, n_{S}$ and $n_{I}$ ). For the second step, the sets of drivers choosing each strategy and thus the equilibrium values of $n_{R}, n_{S}$ and $n_{I}$ are determined given the individual strategy choices derived in step 1 .

\subsection{Driver strategy choice with exogenous traffic}

The first step is to derive an individual driver's choice between strategies $R, S$ and $I$ as a function of $\theta$, the price of information, $\pi$, and traffic conditions $\left(n_{R}, n_{S}, n_{I}\right)$. If a driver is not informed, the choice is restricted to strategies $R$ and $S$. The preference ranking between $R$ and $S$ is described by the condition:

$$
R \succ S \Leftrightarrow p U\left(t_{R}^{+}\left(n_{R}\right) ; \theta\right)+(1-p) U\left(t_{R}^{-} ; \theta\right)>p U\left(t_{S}\left(n_{S}+n_{I}\right) ; \theta\right)+(1-p) U\left(t_{S}\left(n_{S}\right) ; \theta\right),
$$

where the expected utilities of $R$ and $S$ are as given in Table 2. Rearranging terms, this condition can be written in terms of the difference in utilities:

$$
R \succ S \Leftrightarrow \psi_{R S}\left(\theta ; p, n_{R}, n_{S}, n_{I}\right) \equiv \begin{aligned}
& p\left[U\left(t_{R}^{+}\left(n_{R}\right) ; \theta\right)-U\left(t_{S}\left(n_{S}+n_{I}\right) ; \theta\right)\right] \\
& +(1-p)\left[U\left(t_{R}^{-} ; \theta\right)-U\left(t_{S}\left(n_{S}\right) ; \theta\right)\right]
\end{aligned}>0 .
$$

Naturally, for given $n_{I}$ this condition does not depend on the price of information - although if $\pi$ is small enough a driver may prefer strategy $I$ to either $R$ or $S$. The properties of the preference ranking between $R$ and $S$ are described in:

Lemma 2 Under Assumptions 1-4:

(a) There exists a unique risk aversion threshold $\hat{\theta}_{R S}\left(p, n_{R}, n_{S}, n_{I}\right)$ such that $R \succ S \Leftrightarrow \theta<\hat{\theta}_{R S}\left(p, n_{R}, n_{S}, n_{I}\right)$.

(b) For $\hat{\theta}_{R S}<\infty, \hat{\theta}_{R S}$ is decreasing in $p$ and $n_{R}$, and increasing in $n_{S}$ and $n_{I}$.

Proof. See Appendix 9.

Lemma 2 is the counterpart to Lemma 1 for Zero information. The threshold $\hat{\theta}_{R S}$ is a decreasing function of $p$ and $n_{R}$ for the same reason that $\tilde{\theta}^{Z}$ is decreasing. $\hat{\theta}_{R S}$ is increasing in $n_{S}$ and $n_{I}$ because the safe route becomes less attractive. 
The preference ranking between strategies $R$ and $I$ is described by the condition:

$$
R \succ I \Leftrightarrow p U\left(t_{R}^{+}\left(n_{R}\right) ; \theta\right)+(1-p) U\left(t_{R}^{-} ; \theta\right)>p U\left(t_{S}\left(n_{S}+n_{I}\right)+\pi ; \theta\right)+(1-p) U\left(t_{R}^{-}+\pi ; \theta\right),
$$

which is equivalent to:

$$
R \succ I \Leftrightarrow \psi_{R I}\left(\theta, \pi ; p, n_{R}, n_{S}+n_{I}\right) \equiv \begin{gathered}
p\left[U\left(t_{R}^{+}\left(n_{R}\right) ; \theta\right)-U\left(t_{S}\left(n_{S}+n_{I}\right)+\pi ; \theta\right)\right] \\
+(1-p)\left[U\left(t_{R}^{-} ; \theta\right)-U\left(t_{R}^{-}+\pi ; \theta\right)\right]
\end{gathered}>0 .
$$

Note that this condition depends on the combined numbers of drivers who choose Strategies $S$ and $I, n_{S}+n_{I}$. It does not depend independently on $n_{S}$ and $n_{I}$ because Strategies $R$ and $I$ differ only in the $B a d$ state where drivers choosing Strategies $S$ and $I$ all take route $S$. The properties of the preference ranking between $R$ and $I$ are described in:

Lemma 3 Under Assumptions 1-4:

(a) There exists a unique risk aversion threshold $\hat{\theta}_{R I}\left(\pi, p, n_{R}, n_{S}+n_{I}\right)$ such that $R \succ I \Leftrightarrow \theta<\hat{\theta}_{R I}\left(\pi, p, n_{R}, n_{S}+n_{I}\right)$.

(b) For $\hat{\theta}_{R I}<\infty, \hat{\theta}_{R I}$ is decreasing in $p$ and $n_{R}$, and increasing in $\pi$ and $n_{S}+n_{I}$.

Proof. See Appendix 10.

The comparative statics properties of $\hat{\theta}_{R I}$ are similiar to those of $\hat{\theta}_{R S}$ in Lemma 2. $\hat{\theta}_{R I}$ is increasing in $\pi$ because Strategy $I$ becomes less attractive as the cost of information rises. The preference ranking for the final pair of strategies, $I$ and $S$, is:

$$
I \succ S \Leftrightarrow p U\left(t_{S}\left(n_{S}+n_{I}\right)+\pi ; \theta\right)+(1-p) U\left(t_{R}^{-}+\pi ; \theta\right)>p U\left(t_{S}\left(n_{S}+n_{I}\right) ; \theta\right)+(1-p) U\left(t_{S}\left(n_{S}\right) ; \theta\right),
$$

or

$$
I \succ S \Leftrightarrow \psi_{I S}\left(\theta, \pi ; p, n_{S}, n_{I}\right) \equiv \begin{gathered}
p\left[U\left(t_{S}\left(n_{S}+n_{I}\right)+\pi ; \theta\right)-U\left(t_{S}\left(n_{S}+n_{I}\right) ; \theta\right)\right] \\
+(1-p)\left[U\left(t_{R}^{-}+\pi ; \theta\right)-U\left(t_{S}\left(n_{S}\right) ; \theta\right)\right]
\end{gathered}>0 .
$$

The properties of the preference ranking between $I$ and $S$ are given in

Lemma 4 Under Assumptions 1-4:

(a) There exists a unique risk aversion threshold $\hat{\theta}_{I S}\left(\pi, p, n_{S}, n_{I}\right)$ such that $I \succ S \Leftrightarrow \theta<\hat{\theta}_{I S}\left(\pi, p, n_{S}, n_{I}\right)$.

(b) For $\hat{\theta}_{I S}<\infty, \hat{\theta}_{I S}$ is decreasing in $\pi, p$ and $n_{I}$, independent of $n_{R}$, of ambiguous sign in $n_{S}$, but increasing in $n_{S}$ with $n_{S}+n_{I}$ held fixed.

Proof. See Appendix 11.

To see why threshold $\hat{\theta}_{I S}$ is independent of $n_{R}$ note that users of Strategy $S$ never take route $R$, while users of Strategy $I$ take it only on Good days when travel time is independent of usage.

Figure 4 about here

Figure 4 depicts the combinations of $(\theta, \pi)$ for which drivers choose strategy $R, S$ or $I$ when traffic conditions are fixed. Along the boundary labelled $R \approx S, \psi_{R S}=0$ and drivers are indifferent between $R$ and $S$. The boundary between regions $R$ and $I$ where $R \approx I$, and the boundary between regions $I$ and $S$ where $I \approx S$, are interpreted similarly. The locations of the indifference curves depicted in Figure 4 are described by the following two remarks. 
Remark 1 When $R \succ S$, the curve $R \approx I$ lies below the curve $I \approx S$. When $S \succ R$, the curve $R \approx I$ lies above the curve $I \approx S$.

Proof. Transitivity of preferences implies that, when $R \succ S$ and $I \approx S$, then $R \succ I$ and the curve $R \approx I$ is located at a lower $\pi$. Similarly, when $S \succ R$ and $I \approx S$, then $R \prec I$ and the curve $R \approx I$ is located at a higher $\pi$.

Remark 1 confirms that the decision whether to become informed involves a comparison between $I$ and $R$ for the least risk-averse drivers, and a comparison between $I$ and $S$ for the most risk-averse drivers.

Remark 2 When $\pi=\theta=0, I \succ S$ and either $R \succ I$ or $I \succ R$ depending on the value of $n_{R}$.

Proof. When $\theta=0, U(t ; 0)=-(a+t)$ by Assumption 3. If $\pi=0$ as well, then

$$
\begin{gathered}
\psi_{I S}\left(\theta=0, \pi=0 ; p, n_{S}, n_{I}\right)=(1-p)\left[t_{S}\left(n_{S}\right)-t_{R}^{-}\right]>0, \text { and } \\
\psi_{R I}\left(\theta=0, \pi=0 ; p, n_{R}, n_{S}+n_{I}\right)=p\left[t_{S}\left(n_{S}+n_{I}\right)-t_{R}^{+}\left(n_{R}\right)\right] \gtrless 0 .
\end{gathered}
$$

Lemmas 2, 3 and 4 lead to:

Proposition 5 Under Assumptions 1-4, for any $\left(p, n_{R}, n_{S}, n_{I}\right)$ there exists a unique price $\widehat{\pi}\left(p, n_{R}, n_{S}, n_{I}\right)>0$, a unique risk aversion threshold $\hat{\theta}_{R S}\left(p, n_{R}, n_{S}, n_{I}\right)$ and two functions $\hat{\theta}_{R I}\left(\pi, p, n_{R}, n_{S}+n_{I}\right)$ and $\hat{\theta}_{I S}\left(\pi, p, n_{S}, n_{I}\right)$ respectively increasing and decreasing in $\pi$ such that:

$$
\hat{\theta}_{R I}\left(\widehat{\pi}\left(p, n_{R}, n_{S}, n_{I}\right), p, n_{R}, n_{S}+n_{I}\right)=\hat{\theta}_{I S}\left(\widehat{\pi}\left(p, n_{R}, n_{S}, n_{I}\right), p, n_{S}, n_{I}\right)=\hat{\theta}_{R S}\left(p, n_{R}, n_{S}, n_{I}\right) .
$$

$A$ driver selects strategy $R$ if $\theta<\hat{\theta}_{R S}\left(p, n_{R}, n_{S}, n_{I}\right)$ and $\theta<\hat{\theta}_{R I}\left(\pi, p, n_{R}, n_{S}+n_{I}\right)$; selects $S$ if $\theta>\hat{\theta}_{R S}\left(p, n_{R}, n_{S}, n_{I}\right)$ and $\theta>\hat{\theta}_{I S}\left(\pi, p, n_{S}, n_{I}\right)$, and selects $I$ if $\theta>\hat{\theta}_{R I}\left(\pi, p, n_{R}, n_{S}+n_{I}\right)$ and $\theta<\hat{\theta}_{I S}\left(\pi, p, n_{S}, n_{I}\right)$. For $\hat{\theta}_{R S}<\infty, \hat{\theta}_{R S}$ is decreasing in $p$ and $n_{R}$, and increasing in $n_{S}$ and $n_{I}$. For $\hat{\theta}_{R I}<\infty, \hat{\theta}_{R I}$ is decreasing in $p$ and $n_{R}$, and increasing in $\pi$ and $n_{S}+n_{I}$. For $\hat{\theta}_{I S}<\infty, \hat{\theta}_{I S}$ is decreasing in $\pi, p$ and $n_{I}$, independent of $n_{R}$, of ambiguous sign in $n_{S}$, but increasing in $n_{S}$ with $n_{S}+n_{I}$ held fixed.

\subsection{Driver strategy choice and equilibrium}

Proposition 5 characterizes individual driver strategy choices with Costly information for given traffic conditions. The analysis now proceeds to the derivation of equilibrium values of $n_{R}^{C}, n_{S}^{C}$ and $n_{I}^{C}$ as functions of $\pi$. The first step is to establish a critical price for information above which no driver chooses to be informed.

Proposition 6 Under Assumptions 1-4, for any probability $p \in(0,1)$ and any $N>0$, there exists a unique price $\pi^{C}(p, N)$, a unique risk aversion threshold $\theta_{0}^{C}(p, N)$, and a unique traffic equilibrium $\left(n_{R 0}^{C}(p, N), n_{S 0}^{C}=\right.$ $\left.N-n_{R 0}^{C}(p, N), n_{I 0}^{C}=0\right)$ such that the three indifference curves $R \approx S, R \approx I$ and $I \approx S$ cross at $\left(\theta_{0}^{C}, \pi^{C}\right)$. Functions $\theta_{0}^{C}(p, N)$ and $n_{R 0}^{C}(p, N)$ are decreasing in $p$.

Proof. See Appendix 12.

Proposition 6 establishes the existence of a critical price for information, $\pi^{C}(p, N)$, at and above which no driver chooses to be informed, and the equilibrium with Costly information is therefore the same as with No information. Consequently, $\theta_{0}^{C}(p, N)=\tilde{\theta}^{Z}\left(p, n_{R}^{Z}\right)$. It remains to establish existence of equilibrium when $\pi<\pi^{C}(p, N)$. 
Proposition 7 Under Assumptions 1-4, for any probability $p \in(0,1)$, any $N>0$ and any price $\pi \geq 0$, there exists a unique equilibrium $n_{R}^{C}(\pi, p, N), n_{S}^{C}(\pi, p, N), n_{I}^{C}(\pi, p, N)=N-n_{R}^{C}(\pi, p, N)-n_{S}^{C}(\pi, p, N)$. If $\pi \geq \pi^{C}(p, N)$, the equilibrium is as described in Proposition 6 . If $\pi<\pi^{C}(p, N)$, there exist unique risk aversion thresholds, $\theta_{A}(\pi, p, N)$ and $\theta_{B}(\pi, p, N)>\theta_{A}(\pi, p, N)$, such that a driver with $\theta<\theta_{A}(\pi, p, N)$ selects Strategy $R$, a driver with $\theta \in\left(\theta_{A}(\pi, p, N), \theta_{B}(\pi, p, N)\right)$ selects Strategy $I$, and a driver with $\theta>\theta_{B}(\pi, p, N)$ selects Strategy $S$. The comparative statics properties of $n_{R}^{C}, n_{S}^{C}, n_{I}^{C}, \theta_{A}$ and $\theta_{B}$ are given in Table 5 .

Proof. See Appendix 13.

Comparative statics properties of the equilibrium shown in Table 5 are derived in Appendix 14.

\begin{tabular}{|c|c|c|c|}
\hline & $\pi$ & $p$ & $N$ \\
\hline$n_{R}$ & $>0$ & $<0$ & $\in(0,1)$ \\
\hline$n_{S}$ & $?$ & $>0$ & $?$ \\
\hline$n_{I}$ & $?$ & $?$ & $?$ \\
\hline$\theta_{A}$ & $>0$ & $<0$ & $?$ \\
\hline$\theta_{B}$ & $?$ & $<0$ & $?$ \\
\hline
\end{tabular}

Table 5: Comparative statics properties of Costly information equilibrium with risk-averse drivers

Comparison of Table 5 with Table 4 reveals three differences in the properties of equilibria with risk-averse drivers and risk-neutral drivers. First, if the cost of information is positive, sufficiently risk-averse drivers eschew buying it and adopt strategy $S$ instead. By contrast, if drivers are risk neutral none choose strategy $S$ if information is relatively cheap. Second, $n_{S}$ depends on $N$ whereas with risk neutrality it is independent of $N$. Third, several of the comparative statics properties are ambiguous in sign including all three of the derivatives for $n_{I}$. Indeed, it is not possible to rule out that the number of drivers who purchase information is a (locally) increasing function of the price although it seems highly unlikely that this will be the case.

Given Proposition 6 and Proposition 7 it is possible to draw Figure 5, which is the counterpart to Figure 4 with $\left(n_{R}^{C}, n_{S}^{C}, n_{I}^{C}\right)$ set at their equilibrium values. Individual preference rankings for the information regimes are:

$$
\left\{\begin{array}{l}
R \succ S \Leftrightarrow \varphi_{R S}(\theta, \pi ; p, N)>0, \\
R \succ I \Leftrightarrow \varphi_{R I}(\theta, \pi ; p, N)>0 \\
I \succ S \Leftrightarrow \varphi_{I S}(\theta, \pi ; p, N)>0
\end{array}\right.
$$

where

$$
\left\{\begin{array}{c}
\varphi_{R S}(\theta, \pi ; p, N)=\psi_{R S}\left(\theta ; p, n_{R}^{C}(\pi, p, N), n_{S}^{C}(\pi, p, N), n_{I}^{C}(\pi, p, N)\right), \\
\varphi_{R I}(\theta, \pi ; p, N)=\psi_{R I}\left(\theta, \pi ; p, n_{R}^{C}(\pi, p, N), n_{S}^{C}(\pi, p, N)+n_{I}^{C}(\pi, p, N)\right), \\
\varphi_{I S}(\theta, \pi ; p, N)=\psi_{I S}\left(\theta, \pi ; p, n_{S}^{C}(\pi, p, N), n_{I}^{C}(\pi, p, N)\right) .
\end{array}\right.
$$

Figure 5 about here

The equilibrium threshold for $\pi \geq \pi^{C}(p, N)$ is $\theta_{0}^{C}(p, N)=\hat{\theta}_{R S}\left(p, n_{R 0}^{C}, n_{S 0}^{C}, 0\right)$, and for $\pi<\pi^{C}(p, N)$ the thresholds are:

$$
\left\{\begin{array}{l}
\theta_{A}(\pi, p, N)=\hat{\theta}_{R I}\left(\pi, p, n_{R}^{C}(\pi, p, N), n_{S}^{C}(\pi, p, N)+n_{I}^{C}(\pi, p, N)\right), \\
\theta_{B}(\pi, p, N)=\hat{\theta}_{I S}\left(\pi, p, n_{S}^{C}(\pi, p, N), n_{I}^{C}(\pi, p, N)\right) .
\end{array}\right.
$$

In the limit as $\pi \rightarrow 0, \theta_{B} \rightarrow \infty$. Usage of the two routes is continuous at $\pi=0$, and hence travel times, expected utilities and $\mathrm{CVs}$ are continuous as well. 


\subsection{Welfare effects of Costly information}

Four groups of drivers must be distinguished in assessing the welfare effects of Costly information. As shown in Figure 6 , in order of increasing risk aversion these are: drivers who choose strategy $R$ in both the No information and the Costly information regimes, drivers who choose $R$ with No information and $I$ with Costly information, drivers who choose $S$ with $N o$ information and $I$ with Costly information, and finally drivers who choose $S$ in both regimes. The four groups will be called $R R, R I, S I$ and $S S$ respectively, and their $C V \mathrm{~s}$ denoted $C V_{R R}^{C}$, $C V_{R I}^{C}$, $C V_{S I}^{C}$ and $C V_{S S}^{C}$. CV is defined by a different equation for each group:

$$
\begin{aligned}
& \begin{array}{r}
p U\left(t_{R}^{+}\left(n_{R}^{C}\right)+C V_{R R}^{C}(\theta) ; \theta\right)+(1-p) U\left(t_{R}^{-}+C V_{R R}\right. \\
=p U\left(t_{R}^{+}\left(n_{R}^{Z}\right) ; \theta\right)+(1-p) U\left(t_{R}^{-} ; \theta\right) .
\end{array} \\
& p U\left(t_{S}\left(n_{S}^{C}+n_{I}^{C}\right)+\pi+C V_{R I}^{C}(\theta) ; \theta\right)+(1-p) U\left(t_{R}^{-}+\pi+C V_{R I}^{C}(\theta) ; \theta\right) \\
& R I\left(\text { if } \theta_{A}<\theta<\theta_{0}^{C}\right) \text { : } \quad=p U\left(t_{R}^{+}\left(n_{R}^{Z}\right) ; \theta\right)+(1-p) U\left(t_{R}^{-} ; \theta\right) \text {. } \\
& \begin{array}{rr}
\quad p U\left(t_{S}\left(n_{S}^{C}+n_{I}^{C}\right)+\pi+C V_{S I}^{C}(\theta) ; \theta\right)+(1-p) \\
=U\left(t_{S}\left(n_{S}^{Z}\right) ; \theta\right) .
\end{array} \\
& \begin{array}{c}
S S\left(\text { if } \theta>\theta_{B}\right): \quad p U\left(t_{S}\left(n_{S}^{C}+n_{I}^{C}\right)+C V_{S S}^{C}(\theta) ; \theta\right)+(1-p) U\left(t_{S}\left(n_{S}^{C}\right)+C V_{S S}^{C}(\theta) ; \theta\right) \\
=U\left(t_{S}\left(n_{S}^{Z}\right) ; \theta\right) .
\end{array}
\end{aligned}
$$

The left-hand side of each equation in (3) is expected utility with Costly information, and the right-hand side is expected utility with $N o$ information. Note that the cost of information, $\pi$, is added to $C V_{R I}^{C}$ and $C V_{S I}^{C}$ for groups $R I$ and $S I$ which purchase information so that their CVs are defined as net of $\pi$. As formalized in Theorem 2 below, $C V_{R R}^{C}(\theta)$ and $C V_{R I}^{C}(\theta)$ are increasing functions of $\theta$, and $C V_{S I}^{C}(\theta)$ and $C V_{S S}^{C}(\theta)$ are decreasing functions of $\theta$. Consequently, $C V$ is highest for drivers who are indifferent between strategies $R$ and $S$ in the No information regime in the same way that $\mathrm{CV}$ for Free information is highest for the indifferent driver. CV is also negative for drivers who are sufficiently risk averse.

Figure 6 about here

The CVs for Costly information and Free information can be ranked by comparing the defining equations in (2) and (3):

$$
\begin{aligned}
& C V_{R R}^{C}(\theta)<C V_{R}^{F}(\theta) \quad \text { since } n_{R}^{C+}>n_{R}^{F+}, \\
& \left.\begin{array}{l}
C V_{R I}^{C}(\theta) \gtrless C V_{R}^{F}(\theta) \\
C V_{S I}^{C}(\theta) \gtrless C V_{S}^{F}(\theta)
\end{array}\right\} \quad \text { depending on } \pi \text { and the relative magnitudes of } t_{S}\left(n_{S}^{C}+n_{I}^{C}\right) \text { and } t_{R}^{+}\left(n_{R}^{F+}\right), \\
& C V_{S S}^{C}(\theta) \gtrless C V_{S}^{F}(\theta) \quad \text { depending on the magnitudes of } t_{R}^{+}\left(n_{R}^{F+}\right), t_{S}\left(n_{S}^{C}+n_{I}^{C}\right), t_{S}\left(n_{S}^{C}\right) \text { and } t_{R}^{-} \text {. }
\end{aligned}
$$

CV for group $R R$ is unambiguously smaller for Costly information than for Free information because fewer drivers shift off route $R$ in the Bad state. But no general ranking is possible for the other three groups. These results are formalized in Theorem 2 which is the counterpart to Theorem 1 for Free information:

Theorem 2 Under Assumptions 1-4: 
(a) The $C V$ for Costly information, $C V^{C}(\theta)$, is an increasing function of risk aversion for the least risk-averse drivers who take route $R$ with No information, and a decreasing function of risk aversion for the most risk-averse drivers who take route $S$ with No information.

(b) When the risk aversion parameter is distributed over $\mathbb{R}^{+}, C V^{C}(\theta)<0$ for the most risk-averse drivers.

(c) $C V^{C}(\theta)<C V^{F}(\theta)$ for the least risk-averse drivers who take route $R$ in both the No information and Costly information regimes. For other drivers the ranking of $C V^{C}(\theta)$ and $C V^{F}(\theta)$ is ambiguous.

Proof. Part (a) is proved in Appendix 7, and Part(b) is proved in Appendix 8. Part (c) was proved in the text.

\section{Private information}

As the cost of information rises towards $\pi^{C}(p, N)$ the number of drivers who purchase information approaches zero and information effectively becomes private in the sense that only a few drivers exploit it. As explained in Section 2, the Costly information equilibrium approaches the equilibrium with No information and the CV for Costly information (gross of the cost) approaches the CV for Private information. The CVs of Groups $R$ and $S$ for Private information, $C V_{R}^{I}(\theta)$ and $C V_{S}^{I}(\theta)$ respectively, are defined by the conditions:

$$
\begin{array}{cc}
R\left(\text { if } \theta<\theta_{R S}\right): & p U\left(t_{S}\left(n_{S}^{Z}\right)+C V_{R}^{I}(\theta) ; \theta\right)+(1-p) U\left(t_{R}^{-}+C V_{R}^{I}(\theta) ; \theta\right)= \\
& p U\left(t_{R}^{+}\left(n_{R}^{Z}\right) ; \theta\right)+(1-p) U\left(t_{R}^{-} ; \theta\right) . \\
\left.S \text { (if } \theta>\theta_{R S}\right): & p U\left(t_{S}\left(n_{S}^{Z}\right)+C V_{S}^{I}(\theta) ; \theta\right)+(1-p) U\left(t_{R}^{-}+C V_{S}^{I}(\theta) ; \theta\right)= \\
U\left(t_{S}\left(n_{S}^{Z}\right) ; \theta\right) .
\end{array}
$$

Since $t_{R}^{+}\left(n_{R}^{Z}\right)>t_{S}\left(n_{S}^{Z}\right), C V_{R}^{I}(\theta)>0$, and since $t_{S}\left(n_{S}^{Z}\right)>t_{R}^{-}, C V_{S}^{I}(\theta)>0$. The CV for Private information is therefore unambiguously positive. Given Assumption 3,CV $V_{R}^{I}(\theta)$ is an increasing function of $\theta$, and $C V_{S}^{I}(\theta)$ is a decreasing function of $\theta$ so that, once again, $\mathrm{CV}$ is highest for drivers with risk aversion $\theta_{R S}$ who are indifferent between strategies $R$ and $S$ with No information.

By comparing (4) with (2) and (3), it is clear that the CV for Private information is larger than the CV for either Free information or Costly information:

$$
\begin{aligned}
& C V_{R}^{I}(\theta)>C V_{R}^{F}(\theta) \text { and } C V_{S}^{I}(\theta)>C V_{S}^{F}(\theta) \text { since } t_{S}\left(n_{S}^{Z}\right)<t_{S}\left(n_{S}^{F+}\right)=t_{R}^{+}\left(n_{R}^{F+}\right), \\
& C V_{R}^{I}(\theta)>C V_{R R}^{C}(\theta) \text { and } C V_{R}^{I}(\theta)>C V_{R I}^{C}(\theta) \text { since } t_{S}\left(n_{S}^{Z}\right)<t_{S}\left(n_{S}^{C}+n_{I}^{C}\right)<t_{R}^{+}\left(n_{R}^{C}\right), \\
& C V_{S}^{I}(\theta)>C V_{S I}^{C}(\theta) \text { and } C V_{S}^{I}(\theta)>C V_{S S}^{C}(\theta) \text { since } t_{S}\left(n_{S}^{Z}\right)<t_{S}\left(n_{S}^{C}+n_{I}^{C}\right) \text { and } t_{R}^{-}<t_{S}\left(n_{S}^{C}\right) .
\end{aligned}
$$

These results are formalized in

Theorem 3 Under Assumptions 1-4:

(a) The $C V$ for Private information is positive for all drivers: $C V^{I}(\theta)>0$.

(b) The $C V$ for Private information exceeds the $C V$ for either Free information or Costly information: $C V^{I}(\theta)>$ $C V^{F}(\theta)$ and $C V^{I}(\theta)>C V^{C}(\theta)$.

(c) The $C V$ for Private information is an increasing function of risk aversion for the least risk-averse drivers who take route $R$ with No information, and a decreasing function of risk aversion for the most risk-averse drivers who take route $S$ with No information.

Proof. Parts (a) and (b) are proved in the text. Part (c) is proved in Appendix 7. 
Private information is more valuable to an individual than is Costly information or Free information because the benefits from selecting the quicker route diminish as more drivers exploit the information. Diminishing returns of this sort have been identified in a number of studies of ATIS with exogenous or endogenous market penetration (e.g., Emmerink, 1998; Yang, 1998). In the model here the negative external effects of information arise not only because the route choice decisions of informed drivers raise travel times for other informed drivers, but also because they contribute to uncertainty in travel times. This impact is especially pernicious for the most risk-averse drivers who try to avoid uncertainty by sticking to the "safe" route. However, uninformed and less risk-averse drivers who take the risky route benefit when informed drivers switch to the safer route on $B a d$ days.

An interesting property of the model is that the CV for Private information for the drivers with risk aversion $\theta_{R S}$ who value it most is strictly positive even in the limit as the probability of $\mathrm{Bad}$ days decreases to zero. This result is formalized in:

Proposition 8 If Assumption 3 holds, and the risk-aversion parameter is distributed over $\mathbb{R}^{+}$, then as the probability of Bad days decreases to zero the maximum $C V$ for Private information approaches from above a limiting value of $\operatorname{Min}\left(t_{R}^{+}(N)-t_{S}(0), t_{S}(0)-t_{R}^{-}\right)>0$.

Proof. See Appendix 15.

An intuitive explanation of sorts for Prop. 8 runs as follows. With No information a driver must choose between Strategy $R$ and Strategy $S$. If he chooses $R$, and Private information then becomes available, he can occasionally save $t_{R}^{+}(N)-t_{S}(0)$ in travel time. (Note that in the limit $p \rightarrow 0$ all drivers take route $R$.) Since this is a recovery from the worst state (a Bad day) an extremely risk-averse driver is willing to pay nearly $t_{R}^{+}(N)-t_{S}(0)$ for the information even though it will be exploited only rarely. If the driver chooses Strategy $S$ instead, and Private information becomes available, he can almost always save $t_{S}(0)-t_{R}^{-}$in travel time. Since this is a gain in the good state (a Good day) the driver is willing to pay nearly $t_{S}(0)-t_{R}^{-}$for the information. Now the driver's expected utility with Private information is a given amount. Hence the driver will effectively choose between strategies $R$ and $S$ according to which willingness to pay is smaller. Hence the actual CV for Private information is the lesser of $t_{R}^{+}(N)-t_{S}(0)$ and $t_{S}(0)-t_{R}^{-}$.

Prop. 8 contrasts with Theorems 1 and 2 which establish that the CVs for Free information and Costly information are negative for very risk-averse individuals. This highlights the contrast between the values of public and private information that was demonstrated by Hirshleifer (1971) in the context of speculative activity. It also suggests that there may be a niche demand for ATIS by highly risk-averse travelers even if travel conditions are fairly predictable.

\section{Numerical example}

The numerical example is representative of a commuting corridor. Travel time functions for the safe route and the risky route on Bad days have a Bureau of Public Roads form:

$$
\left\{\begin{array}{c}
t_{S}\left(n_{S}\right)=\tau_{S}\left[1+\left(\frac{n_{S}}{K_{S}}\right)^{b}\right], \\
t_{R}^{+}\left(n_{R}\right)=\tau_{R}^{+}\left[1+\left(\frac{n_{R}}{K_{R}}\right)^{b}\right],
\end{array}\right.
$$

where $\tau_{S}$ and $\tau_{R}^{+}$are free-flow travel times, $K_{S}$ and $K_{R}$ are capacities, and $b$ is a parameter. For the base case of the example the probability of a $B a d$ day is $p=0.2$ and the number of drivers is $N=10,000$. Other parameter values 
are $\tau_{S}=25 \mathrm{~min} ., \tau_{R}^{+}=25 \mathrm{~min} ., t_{R}^{-}=20 \mathrm{~min} ., K_{S}=10,000$ per hr., $K_{R}=8,000$ per hr. and $b=2$. Drivers have CARA preferences and $\theta$ has a log-logistic distribution with parameter $\bar{\theta}=2$; i.e. $F(\theta)=\theta /(\theta+\bar{\theta})=\theta /(\theta+2)$. (The value of $\bar{\theta}$ is based on estimates in de Palma and Picard (2005).) With these parameter values Assumptions 1-4 are all satisfied.

\subsection{Base-case results}

Summary statistics for the equilibria with the base-case parameterization are listed in Column 1 of Table 6 .

\subsubsection{No information}

For the No information regime $n_{R}^{Z}=6,654$ : about two thirds of drivers (those with $\theta<\theta_{R S}=3.98$ ) choose the risky route. Travel time on the risky route is $t_{R}^{-}=20 \mathrm{~min}$. on Good days, and $t_{R}^{+Z}=42.3 \mathrm{~min}$. on Bad days. Travel time on the safe route is $t_{S}=27.8 \mathrm{~min}$. in both states. Expected travel time is $(1-p) t_{R}^{-}+p t_{R}^{Z+}=24.46$ min. for group $R$, and $27.8 \mathrm{~min}$. for group $S$. Group $S$ therefore incurs more than three min. extra mean travel time for the privilege of travel time reliability.

\subsubsection{Free information}

With Free information all drivers take route $R$ on Good days $\left(n_{R}^{F-}=10,000\right)$ whereas less than half of them do on Bad days $\left(n_{R}^{F+}=4,444\right)$. The 4:5 division of traffic between the two routes is independent of the distribution of risk preferences, and the set of drivers who take Route $R$ on $B a d$ days is indeterminate because travel time is known in advance. All drivers experience the same travel times of $t_{R}^{-}=20 \mathrm{~min}$. on Good days, and $t_{R}^{F+}=t_{S}^{F+}=32.72$ min. on Bad days. Expected travel time is 22.54 min.: a drop from the No information regime of 1.92 min. for Group $R$, and $5.26 \mathrm{~min}$. for Group $S$. Average CVs are $2.60 \mathrm{~min}$. for Group R, and $1.38 \mathrm{~min}$. for Group $S$. Thus, although Group $S$ experiences a much larger average reduction in travel time as per Proposition 4 , Group $S$ benefits less from Free information in terms of CV.

$\mathrm{CV}$ for Free information, $C V^{F}(\theta)$, is plotted in Figure 7. (The other seven curves are discussed later.) Consistent with Theorem $1, C V^{F}(\theta)$ reaches a maximum at $\theta_{R S}=3.98$, and then drops - eventually below zero. Nearly 10 percent of the most risk averse users (with $\theta>18.7$ ) and over a quarter of Group $S$ end up worse off. This is consistent with Theorem 2(b) that sufficiently risk-averse drivers are worse off with Free information.

Figure 7 about here

\subsubsection{Costly information}

The numerical counterpart to Figure 5 for the Costly information regime is shown in Figure 8 . As the cost of information rises from 0 to the choke price of $\pi^{C}(p, N)=C V^{I}\left(\theta_{R S}\right)=5.88$, the number of drivers purchasing information drops steadily to zero. Figure 9 shows how the fractions of drivers in each of the four groups evolve with $\pi$. As expected, the two groups that acquire information ( $R I$ and $S I$ ) decline steadily in size towards zero, whereas Groups $R R$ and $S S$ grow. The fraction of drivers who are worse off decreases with $\pi$ because fewer drivers are informed and there is less variation in travel time on route $S$.

Figure 8 about here 
Figure 10 presents the CV for Costly information by group. The two informed groups always fare better than do the uninformed, and their benefits increase as information becomes cheaper except when the price nears zero. As far as the two uninformed groups, the benefits to Group $R R$ increase monotonically as information becomes cheaper. Group $S S$ gains as well while information is expensive, but it loses out once information becomes cheaper. Nevertheless, the aggregate benefits for the two uninformed groups increase steadily because Group $S S$ shrinks in size. Overall, the pattern is one in which most drivers benefit from information while a small minority of highly risk-averse drivers suffer appreciably.

Figure 9 about here

Figure 7 plots the CVs for Free information, Costly information and Private information as functions of $\pi$. Consistent with Theorems 1,2 and 3 , all three CVs reach their maxima at $\theta=\theta_{R S}$. And consistent with Theorem 3, $C V^{I}(\theta)$ exceeds $C V^{F}(\theta)$ and $C V^{C}(\theta, \pi)$ over the whole range of $\pi$. The behaviour of $C V^{C}(\theta, \pi)$ is rather complex. In the lower range of $\theta, C V^{C}(\theta, \pi)$ is bounded between 0 and $C V^{F}(\theta)$ and decreases monotonically with $\pi$. For larger values of $\theta, C V^{C}(\pi, \theta)>C V^{F}(\theta)$ because highly risk-averse drivers benefit from the fact that with Costly information fewer drivers shift to the safe route on $\operatorname{Bad}$ days. $C V^{C}(\pi, \theta)$ varies non-monotonically with $\pi$ : it rises initially, but eventually declines towards zero as the number of informed drivers diminishes and with it the potential for information to be beneficial. However, part (b) of Theorem 2 guarantees that some drivers are worse off as long as $\pi<\pi^{C}(p, N)$.

Figure 10 about here

\subsubsection{Sensitivity analysis}

Probability of Bad days Figure 11 shows how the effects of Free information evolve with $p$ (the base-case value of $p=0.2$ is marked by the vertical dashed line). Over most of the range $p \in[0,0.5]$ the mean travel time saving and $\mathrm{CV}$ decrease for Group $S$, and increase for Group $R$. In the limit as $p \rightarrow 0$ the effects on Group $R$ decrease to zero as expected. For Group $S$ the travel time reduction is greatest when $p$ is close to zero since Group $S$ is almost always able to save time by taking route $R$ rather than route $S$. As $p$ rises travel time savings for Group $S$ drop, and so does its $C V$ since Group $R$ shifts more frequently to route $S$ and increases travel time there.

Figure 11 about here

Distribution of risk aversion If parameter $\bar{\theta}$ of the log-logistic distribution is reduced to zero, the population degenerates to a set of $N$ identical risk-neutral drivers such as considered in Section 2. Equilibria for this case are shown in Column 2 of Table 6. Compared to the base case, the fraction of drivers taking route $R$ with $N o$ information increases from roughly $2 / 3$ to $4 / 5$, and the difference in travel times on $R$ and $S$ on $B a d$ days is accentuated. This is because drivers are not averse to travel time variability on route $R$. (Chen et al. (2002) obtain a similar result.) Mean travel time savings and CVs all coincide at 3.46 min., and all drivers benefit from Free information by this amount. Figure 12 shows that as parameter $\bar{\theta}$ rises from 0 through the base case value of $\bar{\theta}=2$ and upwards, mean travel time saving and $C V_{R}^{F}$ fall slowly for Group $R$. In contrast, for Group $S$ mean travel time saving rises and $C V_{S}^{F}$ drops rather sharply so that the divergence between travel time saving and CV is much greater than for Group $R$. (To see why, note that with greater risk aversion in the population a larger fraction of drivers take route $S$ with No information. With Free information these drivers enjoy a greater time 
saving from taking route $R$ on Good days, but they also suffer a greater cost from travel time variability on route $S$.) Consistent with this, the fraction of drivers made worse off by Free information rises from zero at $\bar{\theta}=0$ to more than 20 percent at $\bar{\theta}=8$.

Figure 12 about here

Other variations Raising the number of drivers from $N=10,000$ to $N=15,000$ (see Column 3 in Table 6 ) increases the effects of Free information as expected, with the interesting exception that the mean CV for Group $S$ decreases slightly. This is attributable to the fact that with Free information travel time on the safe route varies by $42.36-34.11=8.25 \mathrm{~min}$. compared to only $32.72-27.80=4.92 \mathrm{~min}$. in the base case.

Reducing the capacity of route $R$ on Bad days from $K_{R}=8,000$ to $K_{R}=4,000$ (see Column 4) results in travel times on Bad days with No information similar to the travel times in Column 3. But the benefits from Free information are generally larger because the variation in performance of route $R$ is more pronounced.

In Column 5 parameter $b$ of the travel time function is reduced from 2 to 1 . This increases the divergence between mean travel time reduction and $\mathrm{CV}$ for group $S$, and increases the fraction of drivers made worse off by Free information. This is because with linear travel costs more drivers take route $R$ with No information, and Free information has a bigger impact in shifting drivers to route $S$ on $B a d$ days. Raising parameter $b$ to 4 has the mirror image effect of reducing the fraction made worse off to about 4 percent.

In Column 6 the free-flow travel time on Route $\mathrm{R}, \tau_{R}^{+}$, is reduced from $25 \mathrm{~min}$. to $20 \mathrm{~min}$. to match the free-flow travel time on Good days, $\tau_{R}^{-}$. (This violates the strict inequality $t_{R}^{-}<t_{R}^{+}(0)$ in Assumption 2, but does not invalidate the equilibrium.) Usage of Route $R$ expands considerably in both the No information and Free information regimes. The welfare effects of information are slightly smaller than in the base case, but follow the same pattern. As in Column 5, more drivers take route $R$. But the shift from $R$ to $S$ on Bad days is reduced because $\tau_{R}^{+}$is smaller.

The final "extreme" case (with $N=15,000, b=1, p=0.5$ and $\bar{\theta}=8$ ) combines several of the one-way parameter variations in a direction that accentuates the adverse effects of Free information. Doing so does not affect greatly the average CV for group $R$, but it does result in a negative average CV for Group $S\left(C V_{S}^{F}=-0.21\right)$ and losses for nearly 30 percent of all drivers. This illustrates rather dramatically that even with heavy congestion, a high probability of capacity loss, and a large proportion of highly risk-averse drivers, information is not necessarily very beneficial. Adverse effects of information may be even worse from a welfare-distributional point of view since it is plausible that drivers with a high level of risk aversion (those using the safe route) are also drivers with low income (see empirical findings in de Palma and Picard, 2005). In such a case, information has a regressive effect since it affects negatively low-income people (and positively high-income people). 


\begin{tabular}{|l|l|l|l|l|l|l|l|l|}
\hline & 1 & \multicolumn{1}{|c|}{2} & 3 & 4 & 5 & \multicolumn{1}{c|}{6} & \multicolumn{1}{c|}{7} \\
\hline & $\begin{array}{c}\text { Base } \\
\text { case }\end{array}$ & $\begin{array}{l}\text { Risk } \\
\text { neutral }\end{array}$ & $\begin{array}{c}N= \\
15000\end{array}$ & $\begin{array}{c}K_{R}= \\
4000\end{array}$ & $b=1$ & $\tau_{R}^{+}=20$ & $\begin{array}{l}\text { Extreme } \\
\text { case }\end{array}$ \\
\hline \multicolumn{7}{|c|}{ No information equilibrium } \\
\hline$n_{R}^{Z}$ & 6,654 & 8,000 & 8,963 & 4,665 & 7,144 & 9,117 & 7,551 \\
\hline$n_{S}^{Z}$ & 3,346 & 2,000 & 6,037 & 5,335 & 2,856 & 883 & 7,449 \\
\hline$t_{R}^{Z+}$ & 42.30 & 50.00 & 56.38 & 59.01 & 47.32 & 37.45 & 48.60 \\
\hline$t_{S}$ & 27.80 & 26.00 & 34.11 & 32.11 & 32.14 & 26.60 & 43.62 \\
\hline \multicolumn{7}{|c|}{ Free information equilibrium } \\
\hline$n_{R}^{F+}$ & 4,444 & 4,444 & 6,667 & 2,857 & 4,444 & 5,607 & 6,667 \\
\hline$n_{S}^{F+}$ & 5,556 & 5,556 & 8,333 & 7,143 & 5,556 & 4,393 & 8,333 \\
\hline$t_{R}^{F+}=t_{S}^{F+}$ & 32.72 & 32.72 & 42.36 & 37.76 & 38.89 & 29.82 & 45.83 \\
\hline \multicolumn{7}{|c|}{ Impacts of Free information } \\
\hline $\begin{array}{l}\text { Mean travel time } \\
\text { red'n for group } R\end{array}$ & 1.92 & 3.46 & 2.80 & 4.25 & 1.69 & 1.52 & 1.38 \\
\hline $\begin{array}{l}\text { Mean travel time } \\
\text { red'n for group } S\end{array}$ & 5.26 & 3.46 & 9.64 & 8.56 & 8.36 & 4.63 & 10.71 \\
\hline $\begin{array}{l}\text { Mean } C V_{R}^{F} \\
\text { for group } R\end{array}$ & 2.60 & 3.46 & 4.21 & 5.53 & 2.68 & 2.08 & 2.10 \\
\hline $\begin{array}{l}\text { Mean } C V_{S}^{F} \\
\text { for group } S\end{array}$ & 1.38 & 3.46 & 1.31 & 3.92 & 0.29 & 1.50 & -0.21 \\
\hline $\begin{array}{l}\text { Mean } C V^{F} \\
\text { for all drivers }\end{array}$ & 2.19 & 3.46 & 3.05 & 4.67 & 1.99 & 1.94 & 0.96 \\
\hline Max. $C V^{F}\left(\theta_{R S}\right)$ & 4.26 & 3.46 & 7.24 & 7.75 & 5.32 & 3.73 & 2.69 \\
\hline$\%$ drivers worse off & 9.64 & 0 & 15.06 & 10.62 & 12.60 & 6.38 & 29.85 \\
\hline \multicolumn{7}{|c|}{ Private information } \\
\hline Max. $C V^{I}\left(\theta_{R S}\right)$ & 5.88 & 4.80 & 10.39 & 9.32 & 8.54 & 4.89 & 4.83 \\
\hline
\end{tabular}

Table 6: Effects of Free information: Sensitivity analysis

$$
\dagger N=15,000, b=1, p=0.5, \bar{\theta}=8
$$

Information can be welfare-reducing In the "extreme" case just considered Group $S$ has a negative aggregate $\mathrm{CV}$ for Free information, but its losses are outweighed by the gains for Group $R$. It is also possible for the aggregate $\mathrm{CV}$ of all drivers to be negative. An example can be constructed by relaxing Assumption 4 and considering two groups of drivers: Group 1 with $N_{1}$ risk-neutral individuals $\left(\theta_{1}=0\right)$, and Group 2 with $N_{2}$ infinitely riskaverse individuals $\left(\theta_{2}=\infty\right)$. For a range of parameter values all Group 1 adopts Strategy $R$, and all Group 2 adopts Strategy $S$. With $N_{0}$ information, travel times are then $t_{S}^{Z}=\tau_{S}\left[1+\left(\frac{N_{2}}{K_{S}}\right)^{b}\right], t_{R}^{Z-}=\tau_{R}^{-}$and $t_{R}^{Z+}=$ $\tau_{R}^{+}\left[1+\left(\frac{N_{1}}{K_{R}}\right)^{b}\right]$. Group 1 prefers Route $R$ provided $(1-p) \tau_{R}^{-}+p t_{R}^{Z+}<t_{S}^{Z}$, and Group 2 prefers Route $S$ provided $t_{S}^{Z}<t_{R}^{Z+}$. With Free information the division of drivers between routes on Bad days is determined by the condition that travel times on Routes $R$ and $S$ are equal: $t_{R}^{F+}=t_{S}^{F+}$, or $\tau_{R}^{+}\left[1+\left(\frac{n_{R}^{F+}}{K_{R}}\right)^{b}\right]=\tau_{S}\left[1+\left(\frac{N-n_{R}^{F+}}{K_{S}}\right)^{b}\right]$. Given $t_{S}^{Z}<t_{R}^{Z+}$, this implies $t_{S}^{Z}<t_{S}^{F+}=t_{R}^{F+}<t_{R}^{Z+}$. CVs are $C V_{R}^{F}=. p\left(t_{R}^{Z+}-t_{R}^{F+}\right)>0$ for Group 1, and 
$C V_{S}^{F}=\left(t_{S}^{Z}-t_{S}^{F+}\right)<0$ for Group 2. The total CV for all drivers is $C V_{t o t}=N_{1} p\left(t_{R}^{Z+}-t_{R}^{F+}\right)+N_{2}\left(t_{S}^{Z}-t_{S}^{F+}\right)$. None of the travel times depends on parameter $p$. And the route choice condition for Group $1,(1-p) \tau_{R}^{-}+p t_{R}^{Z+}<t_{S}^{Z}$, is necessarily satisfied in the limit $p \rightarrow 0$ since $\tau_{R}^{-}<t_{S}^{Z}$. Hence $\operatorname{Lim}_{p \rightarrow 0} C V_{\text {tot }}=N_{2}\left(t_{S}^{Z}-t_{S}^{F+}\right)<0$. Thus, if $B a d$ days are sufficiently rare, Free information is welfare-reducing as measured by the sum of compensating variations. By continuity, $C V_{\text {tot }}$ is also negative if Assumption 4 holds and the distribution of $\theta$ is bimodal with peaks at 0 and some large number. $C V_{t o t}$ is also negative if Group 2 has a finite but sufficiently high degree of risk aversion. For example, with $\theta_{2}=120, N_{1}=5,000, N_{2}=5,000$ and other parameters set at their base-case values for the numerical example, $C V_{\text {tot }}=-1,257$ which is -2.8 percent of the aggregate travel time savings from Free information.

\section{Conclusions and extensions}

This paper studies the information-acquisition and route-choice decisions of risk-averse drivers on a simple road network with one "safe" route and one "risky" route. Four information regimes are considered: No information, Free information - which is publicly available at no cost, Costly information - which is publicly available for a fee, and Private information - which is available free to a single individual.

Several general theoretical results are derived. First, it is drivers with intermediate levels of risk aversion who purchase information in order to select the quickest route each day. The least risk-averse drivers remain uninformed and take the risky route every day, while the most risk-averse drivers take the safe route every day. This pattern mirrors a finding of Emmerink et al. (1996) that it is individuals with intermediate demands for travel who gain the most from information because travel is worthwhile for them under some conditions but not others.

Second, Private information is always beneficial to an individual driver relative to No information, and the benefit from Private information exceeds the benefit from Free information or Costly information. Third, Free information and Costly information benefit drivers who are risk neutral or moderately risk averse. But very riskaverse drivers end up worse off even though some of them may be willing to pay for the information. A numerical example suggests that losers are likely to comprise a relatively small fraction of the population, but also that their losses as measured by CV can be comparable to the highest gains of other drivers (cf. Figure 10). Moreover, if a large proportion of the population is highly risk-averse it is possible for the aggregate $\mathrm{CV}$ of drivers to be negative so that the potential losers could - in principle - bribe the potential winners not to implement an ATIS.

The analysis could be extended in various directions. One is to examine more complex road networks. The two-route network with one safe route has the advantage of being analytically tractable. And it is a natural choice to demonstrate the potential drawbacks of public information for highly risk-averse drivers. But real applications of ATIS are likely to involve multiple links and routes. Furthermore, the property of the model that information is most valuable to drivers with intermediate risk aversion is counterintuitive insofar as the benefits from information would seem, a priori, to be greatest for the most risk-averse individuals. In part, this result is driven by the assumptions that demand is inelastic and that there is a safe route with superior "worst-case" properties than the other route.

A second extension is to consider alternatives to the expected utility paradigm. Empirical evidence has been accumulating at least since Allais (1953) that contradicts expected utility theory, and in recent years Prospect Theory and other non-expected utilities have been applied in transportation research. Nevertheless, there are several reasons why these alternatives do not (at least yet) offer a clearly superior paradigm to expected utility theory for analyzing traveler decisions of the sort considered here. See the discussion in de Palma et al. (2008). First, route- 
choice decision-making differs substantially from gambling on monetary values, and it is not obvious that similar behavioral patterns apply in the two contexts. Moreover, it is not clear what is an appropriate reference point for Prospect Theory (Avineri and Prashker, 2004). Second, route-choice decisions are made repeatedly for commuting and other routine trips, and it is plausible that as individuals become familiar with a particular environment their travel decisions will converge towards expected utility maximization. Third, Avineri and Prashker (2005) found that Cumulative Prospect Theory failed to predict route-choice feedback-based decisions observed in laboratory experiments. Yet asymmetry with respect to gains and losses remains potentially relevant to describe route choice decisions. A recent empirical application has been developed by de Lapparent (2009), who imbeds Cumulative Prospect Theory in the Discrete Choice framework.

A third extension is to relax the assumption that travelers know the probability distribution of states and to model learning. Jotisankasa and Polak (2005) review studies of learning in route and departure time choice, and Bonsall (2008) discusses information acquisition and learning. In the model setting here it is drivers with intermediate degrees of risk aversion who have the most to gain from learning the distribution of states on the risky route - either by driving on it themselves or by drawing on other drivers' experience (see Chancelier et al. (2007) for the case of a single driver facing uncertainty and rational learning concerning the unknown probability of good and bad days). The extension involving both learning and congestion would lead to the information cascade problem described in economics (see Bikhchandani et al., 2006). One question that arises is whether provision of information would speed up learning. In theory the answer would appear to be yes, but if information is conveyed in the form of advice it may deter people from learning (Adler, 2001; Chorus et al., 2006).

Finally, the model could be made more practically useful by considering heterogeneity in value of time and idiosyncratic preferences with respect to routes. Such preferences could be estimated using surveys and stated preference methods as in De Palma and Picard (2005). For the purpose of designing and operating ATIS systems this would help to identify different driver categories and the amount and type of information that would be of greatest benefit to them. There is a need for more empirical studies of risk aversion and route-choice decisions in the context of information provision - both in the field and in laboratory settings (see, for example, Helbing (2004), Rapoport et al. (2006) and Hartman (2007)). 


\section{References}

[1] Abdel-Aty, M., R. Kitamura and P.P. Jovanis (1997), "Using stated preference data for studying the effect of advanced traffic information on drivers' route choice", Transportation Research Part C 5C(1), 39-50.

[2] Adler, J.L. (2001), "Investigating the learning effects of route guidance and traffic advisories on route choice behavior", Transportation Research $C$ 2C, 1-14.

[3] Allais, M. (1953), "Le comportement de l'Homme rationnel devant le risque: Critique des postulats et axiomes de l'Ecole Américaine", Econometrica 21(5), 503-546.

[4] Arnott, R., A de Palma and R. Lindsey (1991), "Does providing information to drivers reduce traffic congestion?", Transportation Research Part A 25A, 309-318.

[5] Arnott, R., A. de Palma and R. Lindsey (1996), "Information and usage of free-access congestible facilities", International Economic Review 37(1), 181-203.

[6] Avineri, E. and J.N. Prashker (2004), "Violations of expected utility theory in route-choice stated-preferences: The Certainty Effect and inflating of small probabilities", 83rd Annual Meeting of the Transportation Research Board, Washington, D.C. Conference CD Paper No. 04-2391.

[7] Avineri E. and J. N. Prashker (2005), "Sensitivity to travel time variability", Transportation Research Part C $13 \mathrm{C}, 157-183$.

[8] Bates, J., J. Polak, P. Jones and A. Cook (2001), "The valuation of reliability for personal travel", Transportation Research Part E 37E, 191-229.

[9] Ben-Akiva, M., A. de Palma and I. Kaysi (1991), "Dynamic network models and driver information systems", Transportation Research Part A 25A, 251-266.

[10] Bikhchandani, S., D. A. Hirshleifer and I. Welch (2006), "Information Cascades; Entry Written for the New Palgrave Dictionary of Economics". S. N. Durlauf and L. E. Blume (eds.), The New Palgrave Dictionary of Economics, forthcoming, http://ssrn.com/abstract=844407, Palgrave Macmillan.

[11] Bonsall, P. (2008), "Information systems and other intelligent transport system innovations", D.A. Hensher and K.J. Button (eds.), Handbook of Transport Modelling, 2nd ed., Oxford: Elsevier Science, 559-574.

[12] Brownstone, D. and K.A. Small (2005), "Valuing time and reliability: Assessing the evidence from road pricing demonstrations," Transportation Research Part A 39A, 279-293.

[13] Chancelier, J-P., M. De Lara and A. de Palma (2007), "Risk aversion, road choice, and the one-armed bandit problem", Transportation Science 41(1), 1-14.

[14] Chen, A, Z. Ji and W. Recker (2002), "Travel time reliability with risk-sensitive travelers", Transportation Research Record 1783, 27-33.

[15] Chorus, C.G, E.J.E. Molin and B. van Wee (2006), "Use and effects of Advanced Traveller Information Services (ATIS): A review of the literature", Transport Reviews 26(2), 127-149. 
[16] de Lapparent, M. (2009), "Attitudes toward risk of time losses in travel activity and air route choices", Journal of Intelligent Transport Systems, to appear in a special issue on Risk and Transportation, de Palma and Picard Guest Editors.

[17] de Palma, A. and R. Lindsey (1998), "Information and usage of congestible facilities under different pricing regimes", Canadian Journal of Economics 31(3), 666-692.

[18] de Palma, A. and N. Picard (2005), "Route choice decision under travel time uncertainty", Transportation Research Part A 39A, 295-324.

[19] de Palma, A. and N. Picard (2006), "Equilibria and information provision in risky networks with risk averse drivers", Transportation Science 40(4), 393-408.

[20] de Palma A., M. Ben-Akiva, D. Brownstone, C. Holt, T. Magnac, D. McFadden, P. Moffatt, N. Picard, K. Train, P. Wakker and J. Walker (2008), "Risk, uncertainty and discrete choice models", Marketing Letters, 19, 269-285.

[21] Emmerink, R.H.M. (1998), Information and Pricing in Road Transportation, Berlin: Springer Verlag.

[22] Emmerink, R.H.M., E.T. Verhoef, P. Nijkamp and P. Rietveld (1996), "Endogenizing demand for information in road transport", Annals of Regional Science 30(2), 201-222.

[23] Emmerink, R.H.M., E.T. Verhoef, P. Nijkamp and P. Rietveld (1998), "Information effects in transport with stochastic capacity and uncertainty costs", International Economic Review 39(1), 89-110.

[24] Hartman, J.L. (2007), "The relevance of heterogeneity in a congested route network with tolls: An analysis of two experiments using actual waiting times and monetized time costs" (http://econ.ucsb.edu/ hartman/Research_papers.htm).

[25] Helbing, D. (2004), "Dynamic decision behavior and optimal guidance through information services: Models and experiments". M. Schreckernberg and R. Selten (eds.), Human Behavior and Traffic Networks, Berlin: Springer, 48-95.

[26] Hirshleifer, J. (1971), "The private and social value of information and the reward to inventive activity", American Economic Review 61(4), 561-574.

[27] Jackson, W.B. and J.V. Jucker (1981), "An empirical study of travel time variability and travel choice behavior", Transportation Science 16(4), 460-475.

[28] Jotisankasa, A., and J.W. Polak (2005), "Modelling learning and adaptation in route and departure time choice behaviour: Achievements and prospects". M. Lee-Gosselin and S. Doherty (eds.), Integrated Land-Use and Transport Models, Amsterdam: Elsevier, 133-157.

[29] Kobayashi, K. (1994), "Information, rational expectations and network equilibria - an analytical perspective for route guidance systems", The Annals of Regional Science 28(4), 369-393.

[30] Lam, T.C. and K.A. Small (2001), "The value of time and reliability: Measurement from a value pricing experiment", Transportation Research Part E 37E, 231-251. 
[31] Lo, H.K. and W.Y. Szeto (2002), "A methodology for sustainable traveler information services", Transportation Research Part B 36B(2), 113-130.

[32] Lo, H.K., A. Chen and H. Yang (1999), "Travel time minimization in route guidance with elastic market penetration", Transportation Research Record 1667, 25-32.

[33] Mahmassani, H.S., Chang, G-L. and R. Herman (1986), "Individual Decisions and Collective Effects in a Simulated Traffic System", Transportation Science, 20(4), 258-271.

[34] Mahmassani, H.S. and R. Jayakrishnan (1991), "System performance and user response under real-time information in a congested traffic corridor", Transportation Research Part A 25A(5), 293-308.

[35] Mirchandani, P. and H. Soroush (1987), "Generalized traffic equilibrium with probabilistic travel times and perceptions", Transportation Science 21(3), 133-152.

[36] Rapoport, A., T. Kugler, S. Dugar and E.J. Tisches (2006), "Braess Paradox in the laboratory: Experimental study of route choice in traffic networks with asymmetric costs". T. Kugler, J. Cole Smith, T. Connolly and Y-J. Son (eds.), Decision Modeling and Behavior in Complex and Uncertain Environments, Vol. 21, New York: Springer (DOI 10.1007/978-0-387-77131-1).

[37] Schelling, T.C. (1978), Micromotives and Macrobehavior, New York: Norton.

[38] Small, K.A., C. Winston and J. Yan (2005), "Uncovering the distribution of motorists' preferences for travel time and reliability", Econometrica 73, 1367-1382.

[39] Tatineni, M., D.E. Boyce and P. Michandani (1997), "Experiments to compare deterministic and stochastic network traffic loading models", Transportation Research Record 1607, 16-23.

[40] Varian, H.R. (1992), Microeconomic Analysis, third edition, Norton.

[41] Yang (1998), "Multiple equilibrium behaviors and ATIS with endogenous market penetration", Transportation Research Part B 32B(3), 205-218.

[42] Yin, Y. and H. Ieda (2001a), "Assessing performance reliability of road networks under nonrecurrent congestion", Transportation Research Record 1771, 148-155.

[43] Yin, Y. and H. Ieda (2001b), "Does providing information to drivers improve road network reliability?", paper presented at 9th World Conference on Transport Research, Seoul, South Korea.

[44] Yin, Y., W.H.K. Lam and H. Ieda (2004), "New technology and the modeling of risk-taking behavior in congested road networks", Transportation Research Part C 12C(3-4), 171-192.

[45] Zhang, R. and E.T. Verhoef (2006), "A monopolistic market for advanced traveler information systems and road use efficiency", Transportation Research Part A 40A(5), 424-443. 


\section{Appendixes}

\subsection{Appendix 1: Assumption 3, HARA, CRRA and CARA preferences}

Since CRRA is obtained from HARA simply by setting parameter $a=0$, whereas the transformation from HARA to CARA is more cumbersome, Assumption 3 will be verified separately for HARA and CARA.

\subsubsection{HARA preferences}

$U(t ; \theta) \equiv u=-\frac{(a+t)^{1+\theta}}{1+\theta}$ is differentiable, and $U(t ; 0)=-(a+t)$. For $\theta>0$ and $t>0, U(t ; \theta)$ is negative and strictly concave in $t$. $\frac{\partial U}{\partial \theta}=-\frac{(1+\theta)(a+t)^{1+\theta} \ln (a+t)-(a+t)^{1+\theta}}{(1+\theta)^{2}}=-\frac{(a+t)^{1+\theta}}{1+\theta}\left(\ln (a+t)-\frac{1}{1+\theta}\right)$. Now $(a+t)^{1+\theta}=$ $-(1+\theta) u$, and $\ln (a+t)=\frac{\ln (-(1+\theta) u)}{1+\theta}$. Hence $\phi(u) \equiv \frac{\partial U}{\partial \theta}=u \frac{\ln (-(1+\theta) u)-1}{1+\theta}, \frac{\partial \phi}{\partial u}=\frac{\ln (-(1+\theta) u)}{1+\theta}$, and $\frac{\partial^{2} \phi}{\partial u^{2}}=\frac{1}{(1+\theta) u}<$ 0 for $t>0$. Finally, $\operatorname{Lim}_{\theta \rightarrow \infty} \frac{U\left(t_{2} ; \theta\right)}{U\left(t_{1} ; \theta\right)}=\operatorname{Lim}_{\theta \rightarrow \infty}\left(\frac{a+t_{2}}{a+t_{1}}\right)^{1+\theta}=\infty$ for $t_{2}>t_{1}>0$.

\subsubsection{CARA preferences}

$U(t ; \theta) \equiv u=\frac{1-\exp (\theta t)}{\theta}$ is differentiable and $U(t ; 0)=-t$. For $\theta>0$ and $t>0, U(t ; \theta)$ is negative and strictly concave in $t$. $\frac{\partial U}{\partial \theta}=\frac{-\theta+t \exp (\theta t)+\exp (\theta t)-1}{\theta^{2}}$. Now $\exp (\theta t)=1-\theta u$, and $t=\frac{\ln (1-\theta u)}{\theta}$. Hence $\phi(U) \equiv \frac{\partial U}{\partial \theta}=$ $\frac{-\theta(1-\theta u) \frac{\ln (1-\theta u)}{\theta}+1-(1-\theta u)}{\theta^{2}}=\frac{-(1-\theta u) \ln (1-\theta u)+\theta u}{\theta^{2}} \cdot \frac{\partial \phi}{\partial u}=\frac{\theta \ln (1-\theta u)+\theta-\theta}{\theta^{2}}=\frac{\ln (1-\theta u)}{\theta}$, and $\frac{\partial^{2} \phi}{\partial u^{2}}=-\frac{1}{(1-\theta u)}<0$. Finally, $\operatorname{Lim}_{\theta \rightarrow \infty} \frac{U\left(t_{2} ; \theta\right)}{U\left(t_{1} ; \theta\right)}=\operatorname{Lim}_{\theta \rightarrow \infty} \frac{1-\exp \left(\theta t_{2}\right)}{1-\exp \left(\theta t_{2}\right)}=\operatorname{Lim}_{\theta \rightarrow \infty} \exp \left(\theta\left(t_{2}-t_{1}\right)\right)=\infty$ for $t_{2}>t_{1}>0$.

\subsection{Appendix 2: Comparative statics properties of equilibria with risk-neutral drivers}

\subsubsection{No information regime}

In the No information equilibrium the division of traffic, $n_{R}^{Z}$ and $n_{S}^{Z}$, equalizes expected travel costs between routes: $\mathbb{E} C^{Z}=(1-p) t_{R}^{-}+p t_{R}^{+}\left(n_{R}^{Z}\right)=t_{S}\left(n_{S}^{Z}\right)$, where $n_{S}^{Z}=N-n_{R}^{Z}$. Comparative statics properties of the equilibrium are derived by totally differentiating this condition:

$$
\begin{aligned}
& \frac{\partial n_{R}^{Z}}{\partial p}=\frac{t_{R}^{-}-t_{R}^{+}\left(n_{R}^{Z}\right)}{\frac{\partial t_{S}\left(N-n_{R}^{Z}\right)}{\partial n_{S}}+p \frac{\partial t_{R}^{+}\left(n_{R}^{Z}\right)}{\partial n_{R}}}<0, \frac{\partial n_{R}^{Z}}{\partial N}=\frac{\frac{\partial t_{S}\left(N-n_{R}^{Z}\right)}{\partial n_{S}^{Z}}}{\frac{\partial t_{S}\left(N-n_{R}^{Z}\right)}{\partial n_{S}}+p \frac{\partial t_{R}^{+}\left(n_{R}^{Z}\right)}{\partial n_{R}}} \in(0,1), \\
& \frac{\partial E \cdot C^{Z}}{\partial p}=-\frac{\partial t_{S}\left(N-n_{R}^{Z}\right)}{\partial n_{S}} \frac{\partial n_{R}^{Z}}{\partial p}>0, \frac{\partial E \cdot C^{Z}}{\partial N}=\frac{\partial t_{S}\left(N-n_{R}^{Z}\right)}{\partial n_{S}}\left(1-\frac{\partial n_{R}^{Z}}{\partial N}\right)>0 .
\end{aligned}
$$

\subsubsection{Costly information regime}

Equilibrium when $n_{S}^{C}>0$ If strategies $R, I$ and $S$ are all adopted in the Costly information equilibrium, the numbers in each group, $n_{R}^{C}, n_{S}^{C}$ and $n_{I}^{C}$, are determined by the accounting identity

$$
n_{R}^{C}+n_{S}^{C}+n_{I}^{C}=N
$$

and two equal-cost conditions. First, the expected costs of Strategies $I$ and $R$ must be equal:

$$
\pi+(1-p) t_{R}^{-}+t_{S}\left(n_{S}^{C}+n_{I}^{C}\right)=(1-p) t_{R}^{-}+p t_{R}^{+}\left(n_{R}^{C}\right)
$$


Condition (7) can be written $\pi=p\left(t_{R}^{+}\left(n_{R}^{C}\right)-t_{S}\left(n_{S}^{C}+n_{I}^{C}\right)\right)$ : the cost of information must balance the travel time saving gained by taking Route $S$ rather than Route $R$ on $B a d$ days. Second, the expected costs of Strategies $I$ and $S$ must be equal:

$$
\pi+(1-p) t_{R}^{-}+t_{S}\left(n_{S}^{C}+n_{I}^{C}\right)=(1-p) t_{S}\left(n_{S}^{C}\right)+p t_{S}\left(n_{S}^{C}+n_{I}^{C}\right) .
$$

Condition (8) can be written $\pi=(1-p)\left(t_{S}\left(n_{S}^{c}\right)-t_{R}^{-}\right)$: the cost of information must balance the travel time saving gained by taking Route $R$ rather than Route $S$ on Good days.

Totally differentiating (6), (7) and (8) one obtains

$$
\begin{aligned}
& \frac{\partial n_{R}^{C}}{\partial \pi}=\frac{1}{p\left(\frac{\partial t_{S}\left(n_{S}^{C}+n_{I}^{C}\right)}{\partial n_{S}}+\frac{\partial t_{R}^{+}\left(n_{R}^{C}\right)}{\partial n_{R}}\right)}>0, \frac{\partial n_{R}^{C}}{\partial p}=\frac{t_{S}\left(n_{S}^{C}+n_{I}^{C}\right)-t_{R}^{+}\left(n_{R}^{C}\right)}{p\left(\frac{\partial t_{S}\left(n_{S}^{C}+n_{I}^{C}\right)}{\partial n_{S}}+\frac{\partial t_{R}^{+}\left(n_{R}^{C}\right)}{\partial n_{R}}\right)}<0, \\
& \frac{\partial n_{R}^{C}}{\partial N}=\frac{\frac{\partial t_{S}\left(n_{S}^{C}+n_{I}^{C}\right)}{\partial n_{S}}}{\frac{\partial t_{S}\left(n_{S}^{C}+n_{I}^{C}\right)}{\partial n_{S}}+\frac{\partial t_{R}^{+}\left(n_{R}^{C}\right)}{\partial n_{R}}} \in(0,1) ; \frac{\partial n_{S}^{C}}{\partial \pi}=\frac{1}{(1-p) \frac{\partial t_{S}\left(n_{S}^{C}\right)}{\partial n_{S}}}>0, \frac{\partial n_{S}^{C}}{\partial p}=\frac{t_{S}\left(n_{S}^{C}\right)-t_{R}^{-}}{(1-p) \frac{\partial t_{S}\left(n_{S}^{C}\right)}{\partial n_{S}}}>0, \frac{\partial n_{S}^{C}}{\partial N}=0 ; \\
& \frac{\partial n_{I}^{C}}{\partial \pi}=-\left(\frac{\partial n_{R}^{C}}{\partial \pi}+\frac{\partial n_{S}^{C}}{\partial \pi}\right)<0, \frac{\partial n_{I}^{C}}{\partial p}=-\left(\frac{\partial n_{R}^{C}}{\partial p}+\frac{\partial n_{S}^{C}}{\partial p}\right) \stackrel{s}{=}(1-p)^{2} \frac{\partial t_{S}\left(n_{S}^{C}\right)}{\partial n_{S}}-p^{2}\left(\frac{\partial t_{S}\left(n_{S}^{C}+n_{I}^{C}\right)}{\partial n_{S}}+\frac{\partial t_{R}^{+}\left(n_{R}^{C}\right)}{\partial n_{R}}\right) ; \frac{\partial n_{I}^{C}}{\partial N}= \\
& \frac{\frac{\partial t_{R}^{+}\left(n_{R}^{C}\right)}{\left.\partial n_{R}\right)}}{\frac{\partial t_{S}\left(n_{S}^{C}+n_{I}^{C}\right)}{\partial n_{S}}+\frac{\partial t_{R}^{+}\left(n_{R}^{C}\right)}{\partial n_{R}}} \in(0,1) .
\end{aligned}
$$

Using these derivatives it is readily shown that $\mathbb{E} C^{C}$ is an increasing function of $\pi, p$ and $N$. Note that the derivative $\frac{\partial n_{I}^{C}}{\partial p}$ switches sign from positive to negative as $p$ increases.

Equilibrium when $n_{S}^{C}=0$ If the cost of information is sufficiently small, the condition $I \approx S$ is not satisfied even with $n_{S}^{C}=0$; i.e. $\pi<(1-p)\left(t_{S}(0)-t_{R}^{-}\right)$. If so, Condition (8) is not applicable and the equilibrium is derived using Conditions (6) and (7) with $n_{S}^{C}=0$. The comparative statics properties of the model are the same as for $n_{S}^{C}>0$ except that $\frac{\partial n_{I}^{C}}{\partial p}=-\frac{\partial n_{R}^{C}}{\partial p}=\frac{t_{R}^{+}\left(n_{R}^{C}\right)-t_{S}\left(n_{S}^{C}+n_{I}^{C}\right)}{p\left(\frac{\partial t_{S}\left(n_{S}^{C}+n_{I}^{C}\right)}{\partial n_{S}}+\frac{\partial t_{R}^{+}\left(n_{R}^{C}\right)}{\partial n_{R}}\right)}>0$, so that the ambiguity in the sign of this derivative is eliminated.

\subsection{Appendix 3: Proof of Proposition 2}

Part (a) follows from the inequality $t_{S}(0)>t_{R}^{-}$. For Part (b) define $\Psi\left(n_{R}\right) \equiv t_{R}^{+}\left(n_{R}\right)-t_{S}\left(N-n_{R}\right)$. By Assumption $1, \Psi\left(n_{R}\right)$ is a continuous and strictly increasing function of $n_{R}$. By Assumption $2, \Psi(0)=t_{R}^{+}(0)-t_{S}(N)<0$, and $\Psi(N)=t_{R}^{+}(N)-t_{S}(0)>0$. Hence there exists a unique $n_{R}^{F+} \in(0, N)$ such that $\Psi\left(n_{R}^{F+}\right)=t_{R}^{+}\left(n_{R}^{F+}\right)-$ $t_{S}\left(N-n_{R}^{F+}\right)=0$.

\subsection{Appendix 4: Proof of Lemma 1}

Part (a): Define $\psi\left(p, N_{R} ; \theta\right) \equiv p U\left(t_{R}^{+}\left(N_{R}\right) ; \theta\right)+(1-p) U\left(t_{R}^{-} ; \theta\right)-U\left(t_{S}\left(N-N_{R}\right) ; \theta\right)$. $\psi\left(p, N_{R} ; 0\right)=-\left[p t_{R}^{+}\left(N_{R}\right)+(1-p) t_{R}^{-}\right]+t_{S}\left(N-N_{R}\right)>0$. Given $t_{R}^{+}\left(N_{R}\right)>t_{S}\left(N-N_{R}\right)$ and Assumption 3, $\operatorname{Lim}_{\theta \rightarrow \infty} \psi\left(p, N_{R} ; \theta\right)<0$. Given Assumption $3, \psi(\cdot)$ is a continuous function of $\theta$. Hence there exists at least one $\tilde{\theta}^{Z}$ such that $\psi\left(p, N_{R} ; \tilde{\theta}^{Z}\right)=0$ and hence $R \approx S$. Let Let $\mathbb{E}_{i}$ denote the expectations operator for route $i, i \in\{R, S\}$. Then $\psi=\mathbb{E}_{R} U(t ; \theta)-\mathbb{E}_{S} U(t ; \theta)$, and $\frac{\partial \psi}{\partial \theta}=\mathbb{E}_{R} \frac{\partial U(t ; \theta)}{\partial \theta}-\mathbb{E}_{S} \frac{\partial U(t ; \theta)}{\partial \theta}$. By Assumption $3, \partial U / \partial \theta$ is a strictly concave 
function of $U$. Hence by Jensen's inequality

$$
\left.\frac{\partial \psi\left(p, N_{R} ; \theta\right)}{\partial \theta}\right|_{\theta=\tilde{\theta}^{z}\left(p, N_{R}\right)}<0
$$

This proves that $\tilde{\theta}^{Z}\left(p, N_{R}\right)$ is unique. Furthermore,

$$
\psi\left(p, N_{R} ; \theta\right) \gtrless 0 \text { as } \theta \lessgtr \tilde{\theta}^{Z}\left(p, N_{R}\right) .
$$

Part (b): Consider $p^{\prime}>p$. Since $t_{R}^{+}\left(N_{R}\right)>t_{S}\left(N-N_{R}\right), \psi\left(p^{\prime}, N_{R} ; \tilde{\theta}^{Z}\left(p, N_{R}\right)\right)>0$. Given (9) it follows that $\tilde{\theta}^{Z}\left(p^{\prime}, N_{R}\right)<\tilde{\theta}^{Z}\left(p, N_{R}\right)$; hence $\tilde{\theta}^{Z}\left(p, N_{R}\right)$ is strictly decreasing in $p$.

Now consider $N_{R}>N_{R}$. Since $t_{R}^{+}\left(N_{R}\right)$ is increasing in $N_{R}$ and $t_{S}\left(N-N_{R}\right)$ is decreasing in $N_{R}$, $\psi\left(p, \hat{N}_{R} ; \tilde{\theta}^{Z}\left(p, N_{R}\right)\right)>0$. By similar reasoning it follows that $\tilde{\theta}^{Z}\left(p, N_{R}\right)$ is strictly decreasing in $N_{R}$.

\subsection{Appendix 5: Proof of Proposition 3}

Since the $\operatorname{cdf} F(\theta)$ is continuous and strictly increasing for $\theta \in \mathcal{I}, n(\theta)=N F(\theta)$ defines an increasing relationship, with $n(0)=0$ and $n(\theta) \underset{\theta \rightarrow \infty}{\rightarrow} N$. Furthermore, Lemma 1 implies that $\tilde{\theta}^{Z}\left(p, N_{R}\right)$ defines a decreasing relationship between $\theta$ and $n$, with $n(\theta) \in[0, N]$. The two curves therefore cross exactly once, which defines $n_{R}^{Z}(p)$ and $\tilde{\theta}^{Z}\left(p, N_{R}\right)$. Consider the function

$$
\Omega(p, n ; \theta) \equiv p U\left(t_{R}^{+}(n) ; \theta\right)+(1-p) U\left(t_{R}^{-} ; \theta\right)-U\left(t_{S}(N-n) ; \theta\right)
$$

According to Assumption $2, t_{R}^{-}<t_{R}^{+}(n)$, so $U\left(t_{R}^{+}(n) ; \theta\right)-U\left(t_{R}^{-} ; \theta\right)<0$ and $\frac{\partial \Omega}{\partial p}<0$. Decreasing utility and Assumption 1 imply that $U\left(t_{R}^{+}(n) ; \theta\right)$ is decreasing in $n$ and $U\left(t_{S}(N-n) ; \theta\right)$ is increasing in $n$, so $\frac{\partial \Omega}{\partial n}<0$. Finally, for any relevant $(p, n), \Omega$ is locally decreasing in $\theta$ at point $\left(p, n, \tilde{\theta}_{p}^{Z}(n)\right)$ because, according to Lemma 1 , $\Omega\left(p, n, \tilde{\theta}^{Z}(p, n)\right)=0, \Omega(p, n ; \theta)>0$ for any $\theta<\tilde{\theta}^{Z}(p, n)$ and $\Omega(p, n ; \theta)<0$ for any $\theta>\tilde{\theta}^{Z}(p, n)$. As a result, $\frac{\partial \tilde{\theta}^{Z}\left(p, n_{R}^{Z}\right)}{\partial p}=-\frac{\partial \Omega}{\partial p} / \frac{\partial \Omega}{\partial \theta}<0$, and $\frac{\partial n_{R}^{Z}}{\partial p}=\frac{\partial n_{R}^{Z}}{\partial \theta} \frac{\partial \tilde{\theta}^{Z}\left(p, n_{R}^{Z}\right)}{\partial p}=N f\left(\tilde{\theta}^{Z}\right) \frac{\partial \tilde{\theta}^{Z}\left(p, n_{R}^{Z}\right)}{\partial p}<0$.

\subsection{Appendix 6: Proof of Proposition 4}

Part (a): By Proposition 3, in the No information regime

$$
p U\left(t_{R}^{+}\left(n_{R}^{Z}\right) ; \tilde{\theta}^{Z}\right)+(1-p) U\left(t_{R}^{-} ; \tilde{\theta}^{Z}\right)=U\left(t_{S}\left(N-n_{R}^{Z}\right) ; \tilde{\theta}^{Z}\right) \text { where } \tilde{\theta}^{Z}>0
$$

Given $t_{R}^{+}\left(n_{R}^{Z}\right)>t_{R}^{-}$, it follows that

$$
t_{R}^{+}\left(n_{R}^{Z}\right)>t_{S}\left(N-n_{R}^{Z}\right)
$$

With Free information:

$$
t_{R}^{+}\left(n_{R}^{F+}\right)=t_{S}\left(N-n_{R}^{F+}\right) .
$$

Inequality (12) and equation (13) imply $n_{R}^{F+}<n_{R}^{Z}$.

Part (b): The goal is to show $p t_{R}^{+}\left(n_{R}^{F+}\right)+(1-p) t_{R}^{-}<p t_{R}^{+}\left(n_{R}^{Z}\right)+(1-p) t_{R}^{-}<t_{S}\left(N-n_{R}^{Z}\right)$. The first inequality follows from Part (a). The second inequality follows from equation (11) and $\tilde{\theta}^{Z}>0$.

Part (c): Travel times in the No information and Free information regimes are given in the following table: 


\begin{tabular}{|l|l|l|l|l|}
\hline Row & Regime & Group & Good days & Bad days \\
\hline 1 & No information & $R$ & $t_{R}^{-}$ & $t_{R}^{+}\left(n_{R}^{Z}\right)$ \\
\hline 2 & No information & $S$ & $t_{S}\left(N-n_{R}^{Z}\right)$ & $t_{S}\left(N-n_{R}^{Z}\right)$ \\
\hline 3 & Free information & $R$ and $S$ & $t_{R}^{-}$ & $t_{R}^{+}\left(n_{R}^{F+}\right)$ \\
\hline
\end{tabular}

That travel time variability decreases for Group $R$ follows from Rows 1 and 3 , and $t_{R}^{-}<t_{R}^{+}\left(n_{R}^{F+}\right)<t_{R}^{+}\left(n_{R}^{Z}\right)$. That travel time variability increases for Group $S$ follows from Rows 2 and 3 , and $t_{R}^{-}<t_{S}\left(N-n_{R}^{Z}\right)<t_{R}^{+}\left(n_{R}^{F+}\right)$.

\subsection{Appendix 7: Compensating variation and degree of risk aversion}

Let travel time in the No information regime $(Z)$ be $T_{1}$ on Good days, and $T_{2}$ on $B a d$ days. And let travel time in the Free information $(F)$, Costly information $(C)$, or Private information regime $(I)$ be $T_{3}$ on Good days, and $T_{4}$ on Bad days. The compensating variation $C V^{r}(\theta)$ for information regime $r, r \in\{F, C, I\}$, is defined by the condition

$$
\Psi(\theta) \equiv \mathbb{E} U^{r}\left(T+C V^{r}(\theta) ; \theta\right)-\mathbb{E} U^{Z}(T ; \theta)=0,
$$

or

$$
(1-p) U\left(T_{3}+C V^{r}(\theta) ; \theta\right)+p U\left(T_{4}+C V^{r}(\theta) ; \theta\right)-(1-p) U\left(T_{1} ; \theta\right)-p U\left(T_{2} ; \theta\right)=0 .
$$

Two generic cases cover all the cases considered in the text.

8.7.1 Case 1: $T_{1}=T_{3}<T_{4}<T_{2}$

This case covers $C V_{R}^{F}, C V_{R R}^{C}, C V_{R I}^{C}$ and $C V_{R}^{I}$. CV for these cases is guaranteed to be positive. With $C V^{r}(\theta)>0$, $U_{1}>U_{3}>U_{4}>U_{2}$, and No information induces a mean-preserving spread of utility relative to information. Now $\frac{\partial \psi}{\partial \theta}=\mathbb{E}^{r} \frac{\partial U(t ; \theta)}{\partial \theta}-\mathbb{E}^{Z} \frac{\partial U(t ; \theta)}{\partial \theta}$. By Assumption 3, $\partial U / \partial \theta$ is a strictly concave function of $U$. Hence by Jensen's inequality $\left.\frac{\partial \psi}{\partial \theta}\right|_{C V^{r}(\theta)}>0$. Given $\frac{\partial \psi}{\partial C V^{r}(\theta)}<0$, it follows that $C V^{r}(\theta)$ is an increasing function of $\theta$ for Case 1 .

8.7.2 Case 2: $T_{3}<T_{1}=T_{2} \leq T_{4}$

This case covers $C V_{S}^{F}, C V_{S I}^{C}, C V_{S S}^{C}$ and $C V_{S}^{I}$. CV is not guaranteed to be positive. But whether or not it is positive, after $\mathrm{CV}$ is added to travel times $U_{3}>U_{1}=U_{2}>U_{4}$. Information therefore induces a mean-preserving spread of utility relative to No information. Hence by Jensen's inequality $\left.\frac{\partial \psi}{\partial \theta}\right|_{C V^{r}(\theta)}<0$, and $C V^{r}(\theta)$ is a decreasing function of $\theta$ for Case 2 .

\subsection{Appendix 8: Compensating variation is negative for sufficiently risk-averse drivers}

For brevity let $t^{Z}$ denote (certain) travel time with No information, $t_{G}$ travel time on Good days with information, and $t_{B}>t_{G}$ travel time on $B a d$ days with information. $C V(\theta)$ is defined by the condition

$$
p U\left(t_{B}+C V(\theta) ; \theta\right)+(1-p) U\left(t_{G}+C V(\theta) ; \theta\right)-U\left(t^{Z} ; \theta\right)=0,
$$




$$
G(t ; \theta) \equiv U\left(t^{Z} ; \theta\right)[\underbrace{\frac{p U\left(t_{B}+t ; \theta\right)+(1-p) U\left(t_{G}+t ; \theta\right)}{U\left(t^{Z} ; \theta\right)}}_{\equiv H(t ; \theta)}-1]=0 \text { at } t=C V(\theta) .
$$

If $t<t^{Z}-t_{B}$ then $\operatorname{Lim}_{\theta \rightarrow \infty} H(t ; \theta)=0$, and $\underset{\theta \rightarrow \infty}{\operatorname{Lim}} G(t ; \theta)=\infty$. If $t>t^{Z}-t_{B}$ then $\underset{\theta \rightarrow \infty}{\operatorname{Lim}} H(t ; \theta)=\infty$, and $\operatorname{Lim}_{\theta \rightarrow \infty} G(t ; \theta)=-\infty$. Therefore $\operatorname{Lim}_{\theta \rightarrow \infty} C V(\theta)=t^{Z}-t_{B}$. Now $t^{Z}=t_{S}\left(N-n_{R}^{Z+}\right)$. With Free information, $t_{B}=$ $t_{S}\left(N-n_{R}^{F+}\right)$ and $\operatorname{Lim}_{\theta \rightarrow \infty} C V(\theta)=t_{S}\left(N-n_{R}^{Z+}\right)-t_{S}\left(N-n_{R}^{F+}\right)<0$. With Costly information, $t_{B}=t_{S}\left(N-n_{R}^{C+}\right)$ and $\operatorname{Lim}_{\theta \rightarrow \infty} C V(\theta)=t_{S}\left(N-n_{R}^{Z+}\right)-t_{S}\left(N-n_{R}^{C+}\right)<0$.

\subsection{Appendix 9: Proof of Lemma 2}

Part (a): The proof follows closely the proof of Lemma 1. $R \gtreqless S \Longleftrightarrow \psi_{R S}\left(\theta ; p, n_{R}, n_{S}, n_{I}\right) \gtreqless 0$. If $R \succ S$ for all $\theta \in[0, \infty)$ then $\hat{\theta}_{R S}=0$. If $R \prec S$ for all $\theta \in[0, \infty)$ then $\hat{\theta}_{R S}=\infty$. If neither preference ranking holds for all $\theta$ then, by continuity of $\psi_{R S}$ in $\theta$, there exists at least one $\hat{\theta}_{R S}$ such that $\psi_{R S}\left(\hat{\theta}_{R S} ; \cdot\right)=0$. Now $\psi_{R S}=\mathbb{E}_{R} U(t ; \theta)-\mathbb{E}_{S} U(t ; \theta)$, and $\frac{\partial \psi_{R S}}{\partial \theta}=\mathbb{E}_{R} \frac{\partial U(t ; \theta)}{\partial \theta}-\mathbb{E}_{S} \frac{\partial U(t ; \theta)}{\partial \theta}$. By Assumption $3, \partial U / \partial \theta$ is a strictly concave function of $U$, and hence by Jensen's inequality

$$
\left.\frac{\partial \psi_{R S}}{\partial \theta}\right|_{\theta=\hat{\theta}_{R S}}<0
$$

and $\hat{\theta}_{R S}$ is unique. Furthermore,

$$
\psi_{R S}\left(\theta ; p, n_{R}, n_{S}, n_{I}\right) \gtrless 0 \text { as } \theta \lessgtr \hat{\theta}_{R S}\left(p, n_{R}, n_{S}, n_{I}\right) .
$$

Part (b): Consider $p^{\prime}>p$. Since $t_{R}^{-}<t_{S}\left(n_{S}\right)$ and $t_{R}^{+}\left(n_{R}\right) \geq t_{S}\left(n_{S}+n_{I}\right)$,

$$
\begin{aligned}
& U\left(t_{R}^{-} ; \hat{\theta}_{R S}\left(p, n_{R}, n_{S}, n_{I}\right)\right)>U\left(t_{S}\left(n_{S}\right) ; \hat{\theta}_{R S}\left(p, n_{R}, n_{S}, n_{I}\right)\right), \text { and } \\
& U\left(t_{R}^{+}\left(n_{R}\right) ; \hat{\theta}_{R S}\left(p, n_{R}, n_{S}, n_{I}\right)\right) \leq U\left(t_{S}\left(n_{S}+n_{I}\right) ; \hat{\theta}_{R S}\left(p, n_{R}, n_{S}, n_{I}\right)\right) .
\end{aligned}
$$

Therefore $\psi_{R S}\left(\hat{\theta}_{R S}\left(p, n_{R}, n_{S}, n_{I}\right) ; p^{\prime}, n_{R}, n_{S}, n_{I}\right)<0$. Given (14) it follows that

$$
\hat{\theta}_{R S}\left(p^{\prime}, n_{R}, n_{S}, n_{I}\right)<\hat{\theta}_{R S}\left(p, n_{R}, n_{S}, n_{I}\right) .
$$

This proves that $\hat{\theta}_{R S}$ is decreasing in $p$. The comparative statics properties for $n_{R}, n_{S}$ and $n_{I}$ are derived similarly using the respective inequalities

$$
\begin{aligned}
& \psi_{R S}\left(\hat{\theta}_{R S}\left(p, n_{R}, n_{S}, n_{I}\right) ; p, n_{R}^{\prime}, n_{S}, n_{I}\right)<0 \text { for } n_{R}^{\prime}>n_{R}, \\
& \psi_{R S}\left(\hat{\theta}_{R S}\left(p, n_{R}, n_{S}, n_{I}\right) ; p, n_{R}, n_{S}^{\prime}, n_{I}\right)>0 \text { for } n_{S}^{\prime}>n_{S}, \\
& \psi_{R S}\left(\hat{\theta}_{R S}\left(p, n_{R}, n_{S}, n_{I}\right) ; p, n_{R}, n_{S}, n_{I}^{\prime}\right)>0 \text { for } n_{I}^{\prime}>n_{I} .
\end{aligned}
$$

\subsection{Appendix 10: Proof of Lemma 3}

Part (a): The proof follows the proof of Lemma 2(a) by replacing regime $S$ by regime $I$. 
Part (b): Consider $p^{\prime}>p$. Since $t_{R}^{-}<t_{R}^{-}+\pi$ and $t_{R}^{+}\left(n_{R}\right) \geq t_{S}\left(n_{S}+n_{I}\right)$,

$$
\begin{aligned}
& U\left(t_{R}^{-} ; \hat{\theta}_{R I}\left(\pi, p, n_{R}, n_{S}+n_{I}\right)\right)>U\left(t_{R}^{-}+\pi ; \hat{\theta}_{R I}\left(\pi, p, n_{R}, n_{S}+n_{I}\right)\right), \text { and } \\
& U\left(t_{R}^{+}\left(n_{R}\right) ; \hat{\theta}_{R I}\left(\pi, p, n_{R}, n_{S}+n_{I}\right)\right) \leq U\left(t_{S}\left(n_{S}+n_{I}\right) ; \hat{\theta}_{R I}\left(\pi, p, n_{R}, n_{S}+n_{I}\right)\right) .
\end{aligned}
$$

Hence $\psi_{R I}\left(\hat{\theta}_{R I}\left(\pi, p, n_{R}, n_{S}+n_{I}\right), \pi ; p^{\prime}, n_{R}, n_{S}+n_{I}\right)<0$. The counterpart to Condition (15) is

$$
\psi_{R I}\left(\theta, \pi ; p, n_{R}, n_{S}+n_{I}\right) \gtrless 0 \text { as } \theta \lessgtr \hat{\theta}_{R I}\left(\pi, p, n_{R}, n_{S}+n_{I}\right) .
$$

It follows that

$$
\hat{\theta}_{R I}\left(\pi, p^{\prime}, n_{R}, n_{S}+n_{I}\right)<\hat{\theta}_{R I}\left(\pi, p, n_{R}, n_{S}+n_{I}\right) .
$$

The comparative statics properties for $n_{R}, \pi$ and $n_{S}+n_{I}$ are derived similarly using the respective inequalities

$$
\begin{aligned}
& \psi_{R I}\left(\hat{\theta}_{R I}\left(\pi, p, n_{R}, n_{S}+n_{I}\right), \pi ; p, n_{R}^{\prime}, n_{S}+n_{I}\right)<0 \text { for } n_{R}^{\prime}>n_{R}, \\
& \psi_{R I}\left(\hat{\theta}_{R I}\left(\pi, p, n_{R}, n_{S}+n_{I}\right), \pi^{\prime} ; p, n_{R}, n_{S}+n_{I}\right)>0 \text { for } \pi^{\prime}>\pi, \\
& \psi_{R I}\left(\hat{\theta}_{R I}\left(\pi, p, n_{R}, n_{S}+n_{I}\right), \pi ; p, n_{R}, n_{S}^{\prime}+n_{I}^{\prime}\right)>0 \text { for } n_{S}^{\prime}+n_{I}^{\prime}>n_{S}+n_{I} .
\end{aligned}
$$

\subsection{Appendix 11: Proof of Lemma 4}

Part (a): The proof follows the proof of Lemma 2(a) by replacing regime $R$ by regime $I$.

Part (b): The comparative statics properties for $\pi, p, n_{I}, n_{S}$, and $n_{S}$ with $n_{S}+n_{I}$ held constant are derived using the respective inequalities

$$
\begin{gathered}
\psi_{I S}\left(\hat{\theta}_{I S}\left(\pi, p, n_{S}, n_{I}\right), \pi^{\prime} ; p, n_{S}, n_{I}\right)<0 \text { for } \pi^{\prime}>\pi, \\
\psi_{I S}\left(\hat{\theta}_{I S}\left(\pi, p, n_{S}, n_{I}\right), \pi ; p^{\prime}, n_{S}, n_{I}\right)<0 \text { for } p^{\prime}>p, \\
\psi_{I S}\left(\hat{\theta}_{I S}\left(\pi, p, n_{S}, n_{I}\right), \pi ; p, n_{S}, n_{I}^{\prime}\right)<0 \text { for } n_{I}^{\prime}>n_{I}, \\
\psi_{I S}\left(\hat{\theta}_{I S}\left(\pi, p, n_{S}, n_{I}\right), \pi ; p, n_{S}^{\prime}, n_{I}\right) \gtrless 0 \text { for } n_{S}^{\prime}>n_{S}, \\
\psi_{I S}\left(\hat{\theta}_{I S}\left(\pi, p, n_{S}, n_{I}\right), \pi ; p, n_{S}^{\prime}, n_{I}^{\prime}\right)>\text { for } n_{S}^{\prime}>n_{S} \text { and } n_{S}^{\prime}+n_{I}^{\prime}=n_{S}+n_{I}
\end{gathered}
$$

The last three inequalities are readily established if it assumed that all relevant functions are differentiable. For the third inequality:

$$
\frac{\partial \psi_{I S}}{\partial n_{I}}=p[\underbrace{\frac{\partial U\left(t_{S}\left(n_{S}+n_{I}\right)+\pi ; \theta\right)}{\partial t}-\frac{\partial U\left(t_{S}\left(n_{S}+n_{I}\right) ; \theta\right)}{\partial t}}_{-}] \underbrace{\frac{\partial t_{S}\left(n_{S}+n_{I}\right)}{\partial N_{S}}}_{+}<0,
$$


where the term in square brackets is negative because $U(\cdot)$ is strictly concave in $t$. For the fourth inequality:

$$
\begin{gathered}
\frac{\partial \psi_{I S}}{\partial n_{S}}=p[\underbrace{\frac{\partial U\left(t_{S}\left(n_{S}+n_{I}\right)+\pi ; \theta\right)}{\partial t}-\frac{\partial U\left(t_{S}\left(n_{S}+n_{I}\right) ; \theta\right)}{\partial t}}_{-}] \underbrace{\frac{\partial t_{S}\left(n_{S}+n_{I}\right)}{\partial N_{S}}}_{+} \\
-(1-p) \underbrace{\frac{\partial U\left(t_{S}\left(n_{S}\right) ; \theta\right)}{\partial t}}_{-} \underbrace{\frac{\partial t_{S}\left(n_{S}\right)}{\partial N_{S}}}_{+} \gtrless 0 .
\end{gathered}
$$

For the fifth inequality

$$
\frac{\partial \psi_{I S}}{\partial n_{S}}-\frac{\partial \psi_{I S}}{\partial n_{I}}=-(1-p) \underbrace{\frac{\partial U\left(t_{S}\left(n_{S}\right) ; \theta\right)}{\partial t}}_{-} \underbrace{\frac{\partial t_{S}\left(n_{S}\right)}{\partial N_{S}}}_{+}>0 .
$$

\subsection{Appendix 12: Proof of Proposition 6}

By Proposition 5 , for any $\left(p, n_{R}, n_{S}, n_{I}\right)$ there exists a unique price $\pi=\hat{\pi}\left(p, n_{R}, n_{S}, n_{I}\right)$ and a unique risk aversion threshold $\hat{\theta}_{R S}\left(p, n_{R}, n_{S}, n_{I}\right)$ such that a driver with risk aversion $\hat{\theta}_{R S}$ is indifferent between $R, S$ and $I$. With $\pi=\hat{\pi}, n_{I}=0$. The size of group $R$ is $n_{R}=N F(\theta)$ with $\theta=\hat{\theta}_{R S}\left(p, n_{R}, n_{S}, n_{I}=0\right)$. By Assumption $4, F(\cdot)$ is continuous and strictly increasing in $\theta$ over its support so that $n_{R}$ is a continuous and strictly increasing function of $\theta$, with $n_{R}=0$ at $\theta=0$ and $\underset{\theta \rightarrow \infty}{\operatorname{Lim}} n_{R}=N$. By Lemma $2, \hat{\theta}_{R S}$ is continuous and decreasing with $n_{R}$, and continuous and increasing with $n_{S}$. And by Assumption 4, $\hat{\theta}_{R S}\left(p, n_{R}=N, n_{S}=0, n_{I}=0\right)<\infty$. Hence the pair of equations $n_{R}=N F(\theta)$ and $\theta=\hat{\theta}_{R S}\left(p, n_{R}, n_{S}, n_{I}=0\right)$ has a unique solution $n_{R}=n_{R 0}^{C}(p, N)$ and $\theta=\theta_{0}^{C}(p, N)$ with $n_{R 0}^{C}=N F\left(\theta_{0}^{C}\right)$ and $\theta_{0}^{C}=\hat{\theta}_{R S}\left(p, n_{R 0}^{C}, N-n_{R 0}^{C}, 0\right)$. In addition, $\pi^{C}(p, N)=\hat{\pi}\left(p, n_{R 0}^{C}, N-n_{R 0}^{C}, 0\right)$.

To establish the comparative statics properties for $p$, consider $p^{\prime}>p$. The curve $n=N F(\theta)$ is unchanged while the curve $\theta=\hat{\theta}_{R S}\left(p, n_{R}, n_{S}, n_{I}=0\right)$ is decreasing in $p$ by Lemma 2. Hence $\theta_{0}^{C}\left(p^{\prime}, N\right)<\theta_{0}^{C}(p, N)$ and $n_{R 0}^{C}\left(p^{\prime}, N\right)<n_{R 0}^{C}(p, N)$.

\subsection{Appendix 13: Proof of Proposition 7}

For $\pi=\pi^{C}(p, N)$ the equilibrium is as described in Proposition 6 with no one choosing strategy $I$. The same equilibrium clearly applies for $\pi>\pi^{C}(p, N)$. For $0<\pi<\pi^{C}(p, N)$, all three strategies $R, S$ and $I$ are selected. By Proposition 5 , for any $\left(p, n_{R}, n_{S}, n_{I}\right)$ there exists a unique price $\hat{\pi}\left(p, n_{R}, n_{S}, n_{I}\right)$ and two functions $\hat{\theta}_{R I}\left(\pi, p, n_{R}, n_{S}+n_{I}\right)$ and $\hat{\theta}_{I S}\left(\pi, p, n_{S}, n_{I}\right)$ such that for $\pi<\hat{\pi}, \hat{\theta}_{R I}<\hat{\theta}_{I S}$ and $n_{R}=N F\left(\hat{\theta}_{R I}\right), n_{I}=N\left(F\left(\hat{\theta}_{I S}\right)-F\left(\hat{\theta}_{R I}\right)\right)$ and $n_{S}=N\left(1-F\left(\hat{\theta}_{I S}\right)\right)$. The five unknowns $\left(n_{R}^{C}, n_{S}^{C}, n_{I}^{C}, \hat{\theta}_{R I}, \hat{\theta}_{I S}\right)$ can be solved in two steps. The first step is to solve $n_{R}^{C}$ and $\hat{\theta}_{R I}$. The function $N F(\theta)$ is continuous and strictly increasing in $\theta$ over its support. By Proposition $5, \hat{\theta}_{R I}$ is continuous, decreasing in $n_{R}$, and increasing in $n_{S}+n_{I}$. And $\hat{\theta}_{R I}(\pi, p, N, 0)<\infty$. Hence the pair of equations $n_{R}=N F(\theta)$ and $\theta=\hat{\theta}_{R I}\left(\pi, p, n_{R}, N-n_{R}\right)$ has a unique solution $n_{R}=n_{R}^{C}(\pi, p, N)$ and $\theta=\theta_{A}(\pi, p, N)$ with $n_{R}^{C}=N F\left(\theta_{A}\right)$ and $\theta_{A}=\hat{\theta}_{R I}\left(\pi, p, n_{R}^{C}, N-n_{R}^{C}\right)$.

The second step is to solve $n_{S}^{C}, n_{I}^{C}$, and $\hat{\theta}_{I S}$. Now $\hat{\theta}_{I S} \in\left[\hat{\theta}_{R S}, \infty\right)$ and $n_{S}^{C}+n_{I}^{C}=N-n_{R}^{C}$. The function $n_{S}=N(1-F(\theta))$ is continuous and decreasing from $N-n_{R}^{C}$ to 0 as $\hat{\theta}_{I S}$ increases from $\hat{\theta}_{R S}$ to $\infty$. By Proposition 5, $\hat{\theta}_{I S}$ is a continuous and increasing function of $n_{S}$ with $n_{S}+n_{I}$ held fixed. Hence the pair of equations $n_{S}=N(1-F(\theta))$ and $\theta=\hat{\theta}_{I S}\left(\pi, p, n_{S}, n_{I}=N-n_{R}^{C}-n_{S}\right)$ has a unique solution $n_{S}=n_{S}^{C}(\pi, p, N)$ and $\theta_{B}=\hat{\theta}_{I S}\left(\pi, p, n_{S}^{C}, N-n_{R}^{C}-n_{S}^{C}\right)$. Finally, $n_{I}=n_{I}^{C}(\pi, p, N)=N-n_{R}^{C}-n_{S}^{C}$. 


\subsection{Appendix 14: Comparative statics of Costly information equilibrium}

The five endogenous variables $\left\{n_{R}, n_{S}, n_{I}, \theta_{A}, \theta_{B}\right\}$ are determined by the five equations

$$
\begin{gathered}
n_{R}=N F\left(\theta_{A}\right), \\
n_{I}=N\left(F\left(\theta_{B}\right)-F\left(\theta_{A}\right)\right), \\
n_{S}=N\left(1-F\left(\theta_{A}\right)\right),
\end{gathered}
$$

indifference between Strategies $R$ and $I$ at $\theta=\theta_{A}$ :

$$
p U\left(t_{R}^{+}\left(n_{R}\right) ; \theta_{A}\right)+(1-p) U\left(t_{R}^{-} ; \theta_{A}\right)=p U\left(t_{S}\left(N-n_{R}\right)+\pi ; \theta_{A}\right)+(1-p) U\left(t_{R}^{-}+\pi ; \theta_{A}\right),
$$

and indifference between Strategies $I$ and $S$ at $\theta=\theta_{B}$ :

$$
p U\left(t_{S}\left(N-n_{R}\right)+\pi ; \theta_{B}\right)+(1-p) U\left(t_{R}^{-}+\pi ; \theta_{B}\right)=p U\left(t_{S}\left(N-n_{R}\right) ; \theta_{B}\right)+(1-p) U\left(t_{S}\left(n_{S}\right) ; \theta_{B}\right) .
$$

Variable $n_{I}$ does not appear in eqns. (19) or (20), and hence can be solved ex post using eqn. (17). To simplify notation, index the six travel times numerically in order of increasing magnitude:

$$
\underbrace{t_{R}^{-}}_{t_{1}}<\underbrace{t_{R}^{-}+\pi}_{t_{2}}<\underbrace{t_{S}\left(n_{S}\right)}_{t_{3}}<\underbrace{t_{S}\left(n_{S}+n_{I}\right)}_{t_{4}}<\underbrace{t_{S}\left(n_{S}+n_{I}\right)+\pi}_{t_{5}}<\underbrace{t_{R}^{+}\left(n_{R}\right)}_{t_{6}},
$$

and define $m_{i j} \equiv \frac{\partial U\left(t_{i} ; \theta_{j}\right)}{\partial t}<0, c_{i} \equiv \frac{\partial t_{i}}{\partial n_{i}}>0, v_{i j} \equiv \frac{\partial U\left(t_{i} ; \theta_{j}\right)}{\partial \theta}<0$ and $U_{i j} \equiv U\left(t_{i} ; \theta_{j}\right)<0, i=1, \ldots, 6, j=A, B$. (Note that $c_{1}=c_{2}=0<c_{3} \leq c_{4}=c_{5}$.) Eqns. (16), (18), (19) and (20) can then be written:

$$
\begin{gathered}
n_{R}-N F\left(\theta_{A}\right)=0, \\
n_{S}-N\left(1-F\left(\theta_{B}\right)\right)=0, \\
p\left(U_{6 A}-U_{5 A}\right)+(1-p)\left(U_{1 A}-U_{2 A}\right)=0, \\
p\left(U_{4 B}-U_{5 B}\right)+(1-p)\left(U_{3 B}-U_{2 B}\right)=0 .
\end{gathered}
$$

Equations (21) and (23) are separable in $n_{R}$ and $\theta_{A}$. The total differentials are

$$
\begin{aligned}
& {\left[\begin{array}{cc}
1 & -N f\left(\theta_{A}\right) \\
p\left(m_{5 A} c_{5}+m_{6 A} c_{6}\right) & p\left(v_{6 A}-v_{5 A}\right)+(1-p)\left(v_{1 A}-v_{2 A}\right)
\end{array}\right]\left[\begin{array}{l}
d n_{R} \\
d \theta_{A}
\end{array}\right]=} \\
& {\left[\begin{array}{ccc}
0 & 0 & F\left(\theta_{A}\right) \\
p m_{5 A}+(1-p) m_{2 A} & U_{5}-U_{6}+U_{1}-U_{2} & p m_{5 A} c_{5}
\end{array}\right]\left[\begin{array}{c}
d \pi \\
d p \\
d N
\end{array}\right]}
\end{aligned}
$$

Define $\Delta_{A} \equiv p\left(v_{6 A}-v_{5 A}\right)+(1-p)\left(v_{1 A}-v_{2 A}\right)+p\left(m_{5 A} c_{5}+m_{6 A} c_{6}\right) N f\left(\theta_{A}\right)<0$. The comparative statics effects for $n_{R}$ are:

$$
\frac{\partial n_{R}}{\partial \pi}=\Delta_{A}^{-1}[\underbrace{p m_{5 A}+(1-p) m_{2 A}}_{-}] N f\left(\theta_{A}\right)>0,
$$




$$
\begin{gathered}
\frac{\partial n_{R}}{\partial p}=\Delta_{A}^{-1}[\underbrace{U_{5}-U_{6}+U_{1}-U_{2}}_{+}] N f\left(\theta_{A}\right)<0, \\
\frac{\partial n_{R}}{\partial N}=\Delta_{A}^{-1}\{F\left(\theta_{A}\right)[\underbrace{p\left(v_{6 A}-v_{5 A}\right)+(1-p)\left(v_{1 A}-v_{2 A}\right)}_{-}]+N f\left(\theta_{A}\right) \underbrace{p m_{5 A} c_{5}}_{-}\} \in(0,1) .
\end{gathered}
$$

Given (21), (25) and (26),

$$
\begin{gathered}
\frac{\partial \theta_{A}}{\partial \pi}>0, \text { and } \frac{\partial \theta_{A}}{\partial p}<0, \\
\frac{\partial \theta_{A}}{\partial N}=\Delta_{A}^{-1} p\left[m_{5 A} c_{5}\left(1-F\left(\theta_{A}\right)\right)-m_{6 A} c_{6} F\left(\theta_{A}\right)\right] \stackrel{s}{=} ?,
\end{gathered}
$$

where $\stackrel{s}{=}$ ? indicates that the sign is indeterminate. Equations (22) and (24) can be used to derive the comparative statics for $n_{S}$ and $\theta_{B}$ by substituting in the comparative statics derivatives just derived for $n_{R}$. The total differentials are

$$
\begin{gathered}
{\left[\begin{array}{cc}
1 & N f\left(\theta_{B}\right) \\
(1-p) m_{3 B} c_{3} & p\left(v_{4 B}-v_{5 B}\right)+(1-p)\left(v_{3 B}-v_{2 B}\right)
\end{array}\right]\left[\begin{array}{c}
d n_{S} \\
d \theta_{B}
\end{array}\right]=} \\
{\left[\begin{array}{ccc}
0 & 0 & 1-F\left(\theta_{B}\right) \\
p m_{5 B}+(1-p) m_{2 B} & U_{5}-U_{4}+U_{3}-U_{2} & p\left(m_{5 B} c_{5}-m_{4 B} c_{4}\right) \cdot \\
+p\left(m_{4 B} c_{4}-m_{5 B} c_{5}\right) \frac{d n_{R}}{d \pi} & +p\left(m_{4 B} c_{4}-m_{5 B} c_{5}\right) \frac{d n_{R}}{d p} & \left(1-\frac{d n_{R}}{d N}\right)
\end{array}\right]\left[\begin{array}{c}
d \pi \\
d p \\
d N
\end{array}\right] .}
\end{gathered}
$$

Define $\Delta_{B} \equiv p\left(v_{4 B}-v_{5 B}\right)+(1-p)\left(v_{3 B}-v_{2 B}\right)-(1-p) m_{3 B} c_{3} N f\left(\theta_{B}\right)>0$. The comparative statics derivatives for $n_{S}$ are:

$$
\begin{aligned}
\frac{\partial n_{S}}{\partial \pi}=-\Delta_{B}^{-1} & {[\underbrace{p m_{5 B}+(1-p) m_{2 B}}_{-}+p \underbrace{\left(m_{4 B} c_{4}-m_{5 B} c_{5}\right)}_{+} \underbrace{\frac{\partial n_{R}}{\partial \pi}}_{+}] N f\left(\theta_{B}\right) \stackrel{s}{=} ?, } \\
\frac{\partial n_{S}}{\partial p}=-\Delta_{B}^{-1} & {[\underbrace{U_{5}-U_{4}+U_{3}-U_{2}}_{-}+p \underbrace{p\left(m_{4 B} c_{4}-m_{5 B} c_{5}\right)}_{+} \underbrace{\frac{\partial n_{R}}{\partial p}}_{-}] N f\left(\theta_{B}\right)>0, } \\
\frac{\partial n_{S}}{\partial N}=\Delta_{B}^{-1}\{\underbrace{\left[p\left(v_{4 B}-v_{5 B}\right)+(1-p)\left(v_{3 B}-v_{2 B}\right)\right]}_{+}\left(1-F\left(\theta_{B}\right)\right) & >\underbrace{p\left(m_{5 B} c_{5}-m_{4 B} c_{4}\right)}_{-} \underbrace{\left(1-\frac{\partial n_{R}}{\partial N}\right)}_{+} N f\left(\theta_{B}\right)\}
\end{aligned}
$$

Given (22), (30) and (31)

$$
\frac{\partial \theta_{B}}{\partial \pi} \stackrel{s}{=} ?, \text { and } \frac{\partial \theta_{B}}{\partial p}<0
$$


Given (22) and (32)

$$
\begin{aligned}
& \frac{\partial \theta_{B}}{\partial N}=\Delta_{B}^{-1}[p \underbrace{p \underbrace{\left.m_{5 B} c_{5}-m_{4 B} c_{4}\right)}_{5 B}}_{-} \underbrace{\left(1-\frac{\partial n_{R}}{\partial N}\right)}_{+}-(1-p) \underbrace{m_{3 B} c_{3}}_{-}\left(1-F\left(\theta_{B}\right)\right)] \stackrel{s}{=} ?, \\
& \frac{\partial n_{I}}{\partial \pi}=-(\underbrace{\frac{\partial n_{R}}{\partial \pi}}_{+}+\underbrace{\frac{\partial n_{S}}{\partial \pi}}_{?}) \stackrel{s}{=} ?, \\
& \frac{\partial n_{I}}{\partial p}=-(\underbrace{\frac{\partial n_{R}}{\partial p}}_{-}+\underbrace{\frac{\partial n_{S}}{\partial p}}_{+}) \stackrel{s}{=} ?, \\
& \frac{\partial n_{I}}{\partial N}=1-(\underbrace{\frac{\partial n_{R}}{\partial N}}_{+}+\underbrace{\frac{\partial n_{S}}{\partial N}}_{+}) \stackrel{s}{=} ?
\end{aligned}
$$

\subsection{Appendix 15: Proof of Proposition 8}

The proof has a similar structure to the proof of Lemma 2. By Theorem $3, C V^{I}(\theta)$ is maximal at $\theta=\theta_{R S}$, where $\theta_{R S}$ is defined by the condition of indifference between Strategies $R$ and $S$ :

$$
p U\left(t_{R}^{+}\left(n_{R}^{Z}\right) ; \theta_{R S}\right)+(1-p) U\left(t_{R}^{-} ; \theta_{R S}\right)=U\left(t_{S}\left(N-n_{R}^{Z}\right) ; \theta_{R S}\right),
$$

or

$$
p \frac{U\left(t_{R}^{+}\left(n_{R}^{Z}\right) ; \theta_{R S}\right)}{U\left(t_{S}\left(N-n_{R}^{Z}\right) ; \theta_{R S}\right)}+(1-p) \frac{U\left(t_{R}^{-} ; \theta_{R S}\right)}{U\left(t_{S}\left(N-n_{R}^{Z}\right) ; \theta_{R S}\right)}=1 .
$$

Now $\operatorname{Lim}_{p \rightarrow 0} t_{R}^{+}\left(n_{R}^{Z}\right)=t_{R}^{+}(N), \underset{p \rightarrow 0}{\operatorname{Lim}} t_{S}\left(N-n_{R}^{Z}\right)=t_{S}(0), \underset{p \rightarrow 0}{\operatorname{Lim}} \theta_{R S}=\infty$, and

$\operatorname{Lim}_{p \rightarrow 0} \frac{U\left(t_{R}^{-} ; \theta_{R S}\right)}{U\left(t_{S}\left(N-n_{R}^{Z}\right) ; \theta_{R S}\right)}=\operatorname{Lim}_{p \rightarrow 0} \frac{U\left(t_{R}^{-} ; \theta_{R S}\right)}{U\left(t_{S}(0) ; \theta_{R S}\right)}=0$ since $t_{R}^{-}<t_{S}(0)$. Hence by (33)

$$
p \frac{U\left(t_{R}^{+}\left(n_{R}^{Z}\right) ; \theta_{R S}\right)}{U\left(t_{S}\left(N-n_{R}^{Z}\right) ; \theta_{R S}\right)} \underset{p \rightarrow 0}{\rightarrow} 1
$$

$C V^{I}\left(\theta_{R S}\right)$ is defined by the condition

$$
\begin{gathered}
p U\left(t_{S}\left(N-n_{R}^{Z}\right)+C V^{I}\left(\theta_{R S}\right) ; \theta_{R S}\right)+(1-p) U\left(t_{R}^{-}+C V^{I}\left(\theta_{R S}\right) ; \theta_{R S}\right) \\
-U\left(t_{S}\left(N-n_{R}^{Z}\right) ; \theta_{R S}\right)=0,
\end{gathered}
$$

or 


$$
\begin{gathered}
G\left(t ; p, \theta_{R S}(p)\right) \equiv U\left(t_{S}\left(N-n_{R}^{Z}\right) ; \theta_{R S}\right)[\underbrace{p \frac{U\left(t_{S}\left(N-n_{R}^{Z}\right)+t ; \theta_{R S}\right)}{U\left(t_{S}\left(N-n_{R}^{Z}\right) ; \theta_{R S}\right)}}_{\equiv H\left(t ; p, \theta_{R S}(p)\right)}+ \\
\underbrace{(1-p) \frac{U\left(t_{R}^{-}+t ; \theta_{R S}\right)}{U\left(t_{S}\left(N-n_{R}^{Z}\right) ; \theta_{R S}\right)}}_{\equiv J\left(t ; p, \theta_{R S}(p)\right)}-1]=0 \text { at } t=C V^{I}\left(\theta_{R S}\right) .
\end{gathered}
$$

There are two cases to consider.

8.15.1 Case 1: $t_{S}(0)-t_{R}^{-}<t_{R}^{+}(N)-t_{S}(0)$.

If $t<t_{S}(0)-t_{R}^{-}$, then $\underset{p \rightarrow 0}{\operatorname{Lim}} J(\cdot)=0$. And given $t_{S}(0)+t<t_{S}(0)+t_{R}^{+}(N)-t_{S}(0)=t_{R}^{+}(N), \underset{p \rightarrow 0}{\operatorname{Lim}} H(\cdot)=0$ by (34) and $\operatorname{Lim}_{p \rightarrow 0} G(\cdot)=+\infty$.

If $t>t_{S}(0)-t_{R}^{-}$, then $\underset{p \rightarrow 0}{\operatorname{Lim}} J(\cdot)=+\infty \Longrightarrow \operatorname{Lim}_{p \rightarrow 0} G(\cdot)=-\infty$. Therefore $\operatorname{Lim}_{p \rightarrow 0} C V^{I}\left(\theta_{R S}\right)=t_{S}(0)-t_{R}^{-}$.

8.15.2 Case 2: $t_{R}^{+}(N)-t_{S}(0)<t_{S}(0)-t_{R}^{-}$.

If $t<t_{R}^{+}(N)-t_{S}(0)$, then $\operatorname{Lim}_{p \rightarrow 0} J(\cdot)=0, \underset{p \rightarrow 0}{\operatorname{Lim}} H(\cdot)=0$ by $(34)$ and $\underset{p \rightarrow 0}{\operatorname{Lim}} G(\cdot)=+\infty$.

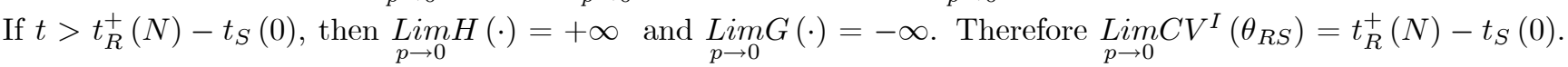

Cases 1 and 2 combined imply $C V^{I}\left(\theta_{R S}\right) \underset{p \rightarrow 0}{\rightarrow} \operatorname{Min}\left(t_{S}(0)-t_{R}^{-}, t_{R}^{+}(N)-t_{S}(0)\right)$. 


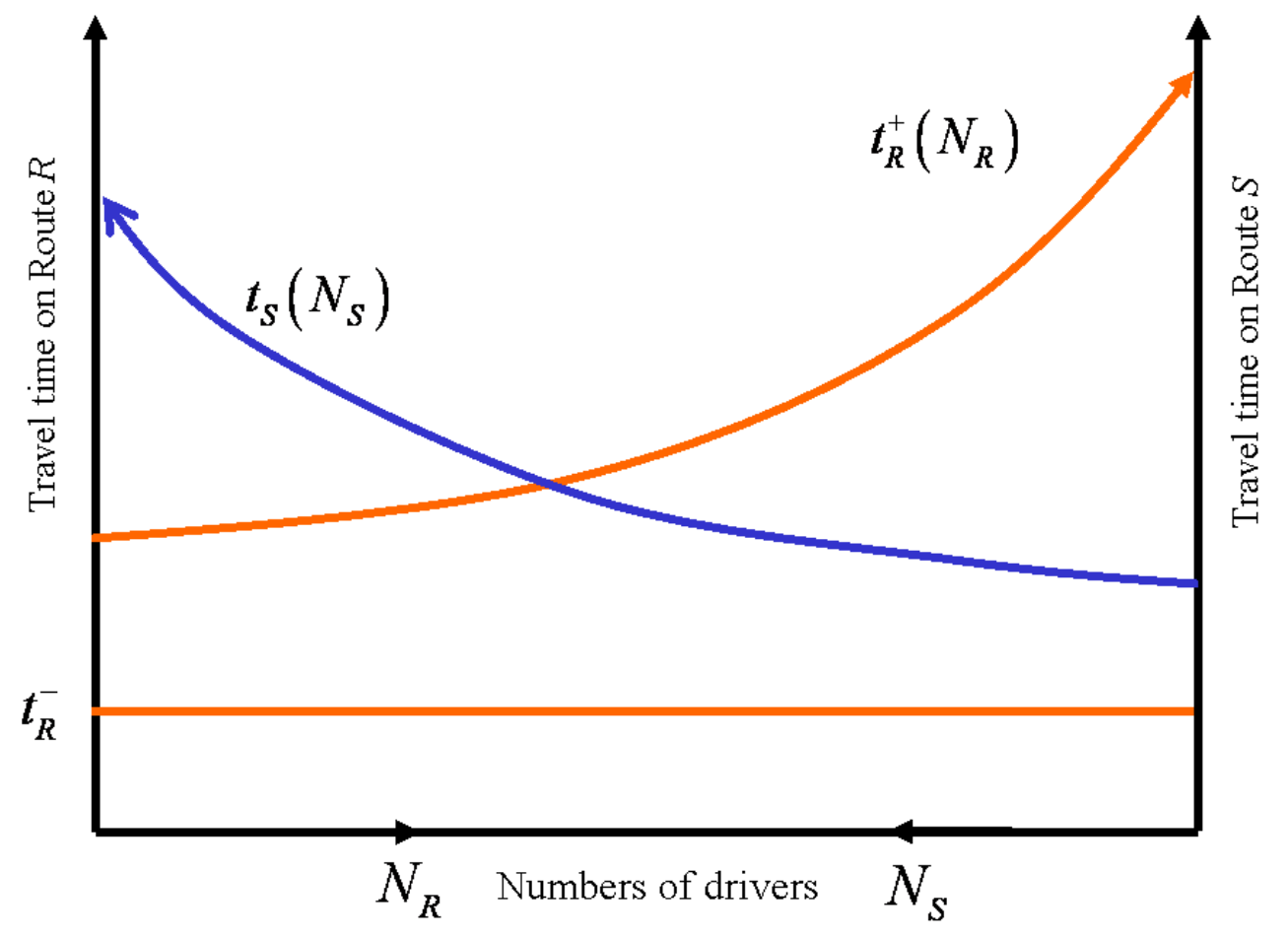

Figure 1: Travel time functions 


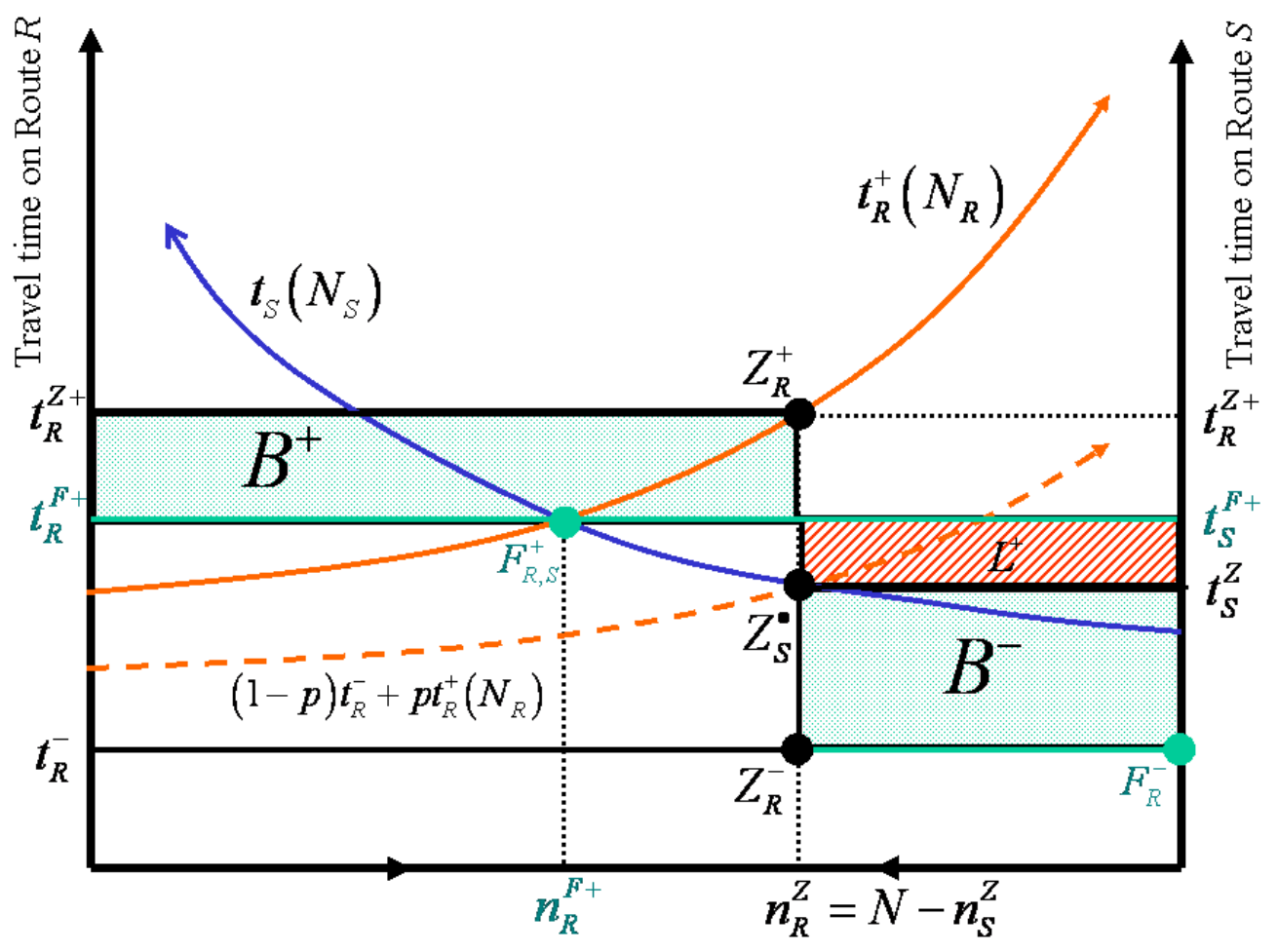

Figure 2: Effects of Free information with risk-neutral drivers and $n_{S}^{C}>0$ 


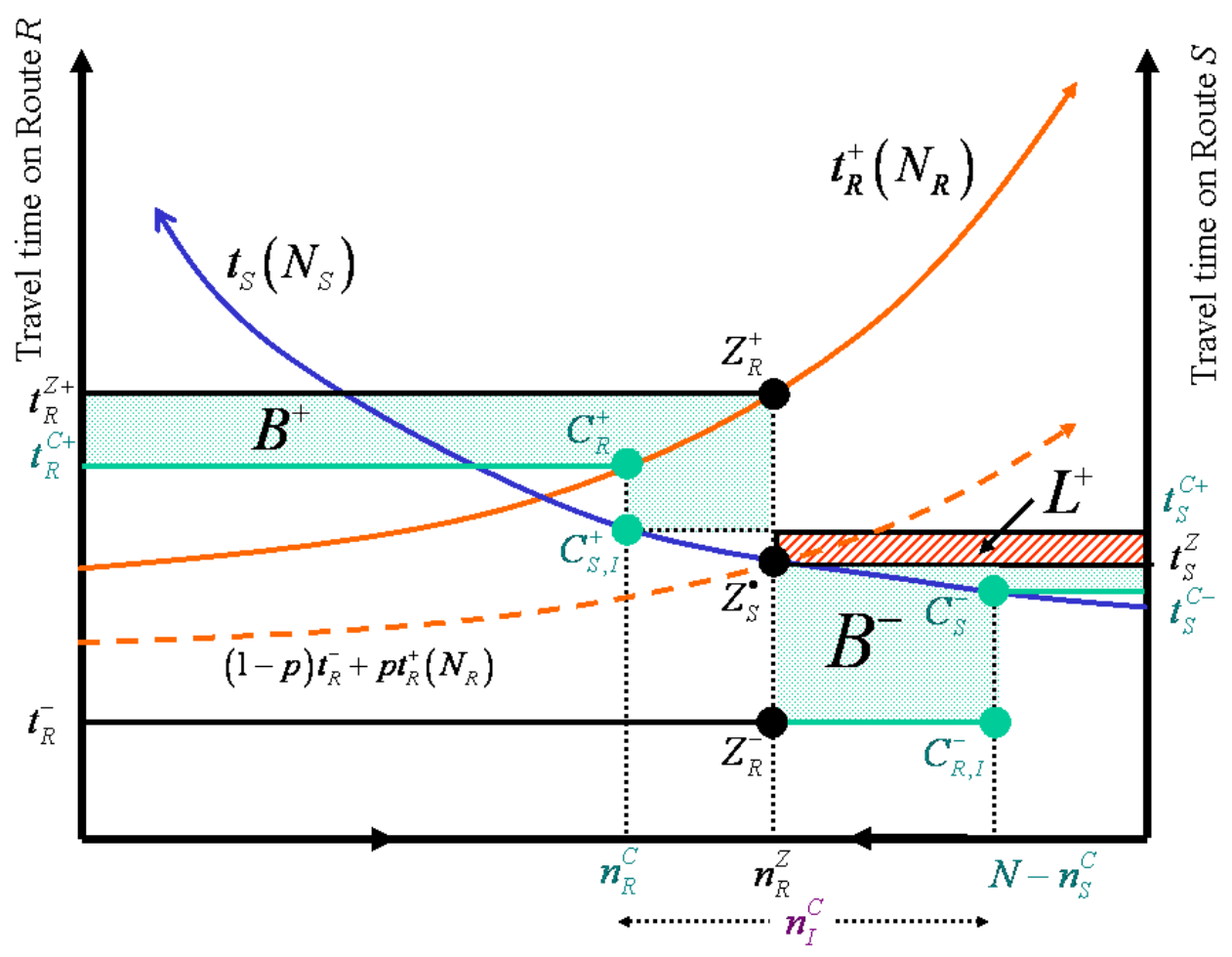

Figure 3: Effects of Costly information with risk-neutral drivers

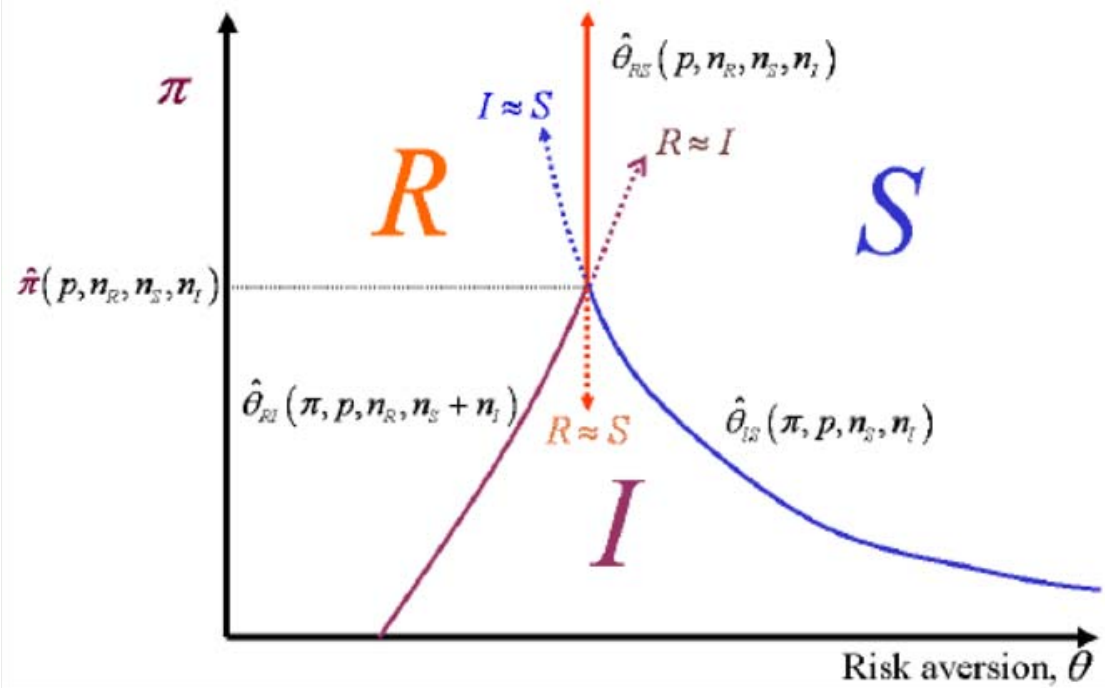

Figure 4: Strategy choice with Costly information 


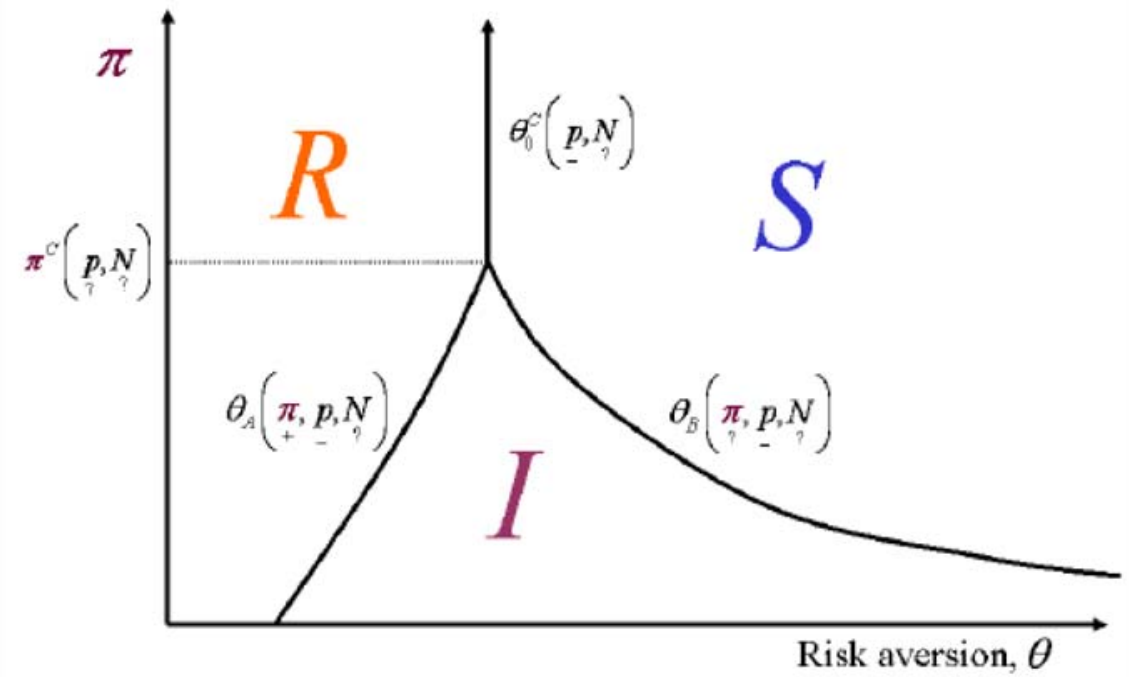

Figure 5: Strategy choice in Costly information equilibrium

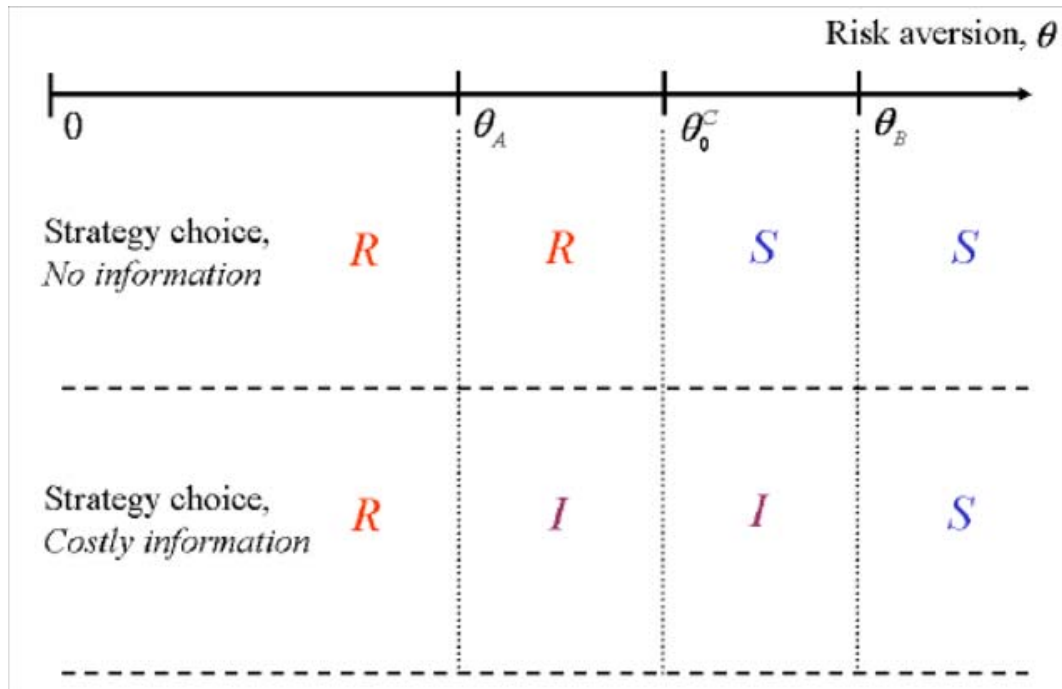

Figure 6: Strategy choices for No information and Costly information regimes 


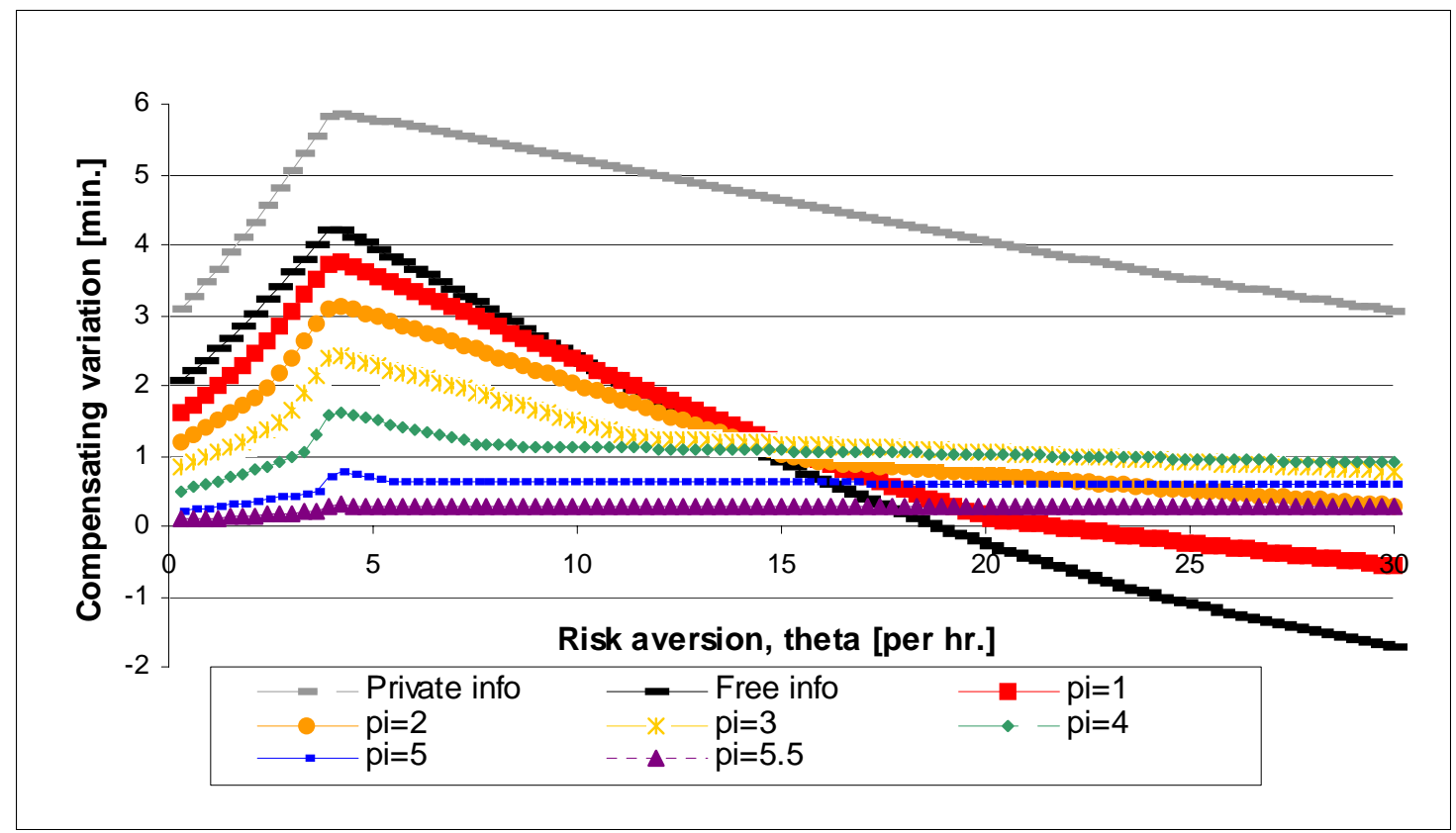

Figure 7: Compensating variations for Free, Costly and Private information in numerical example

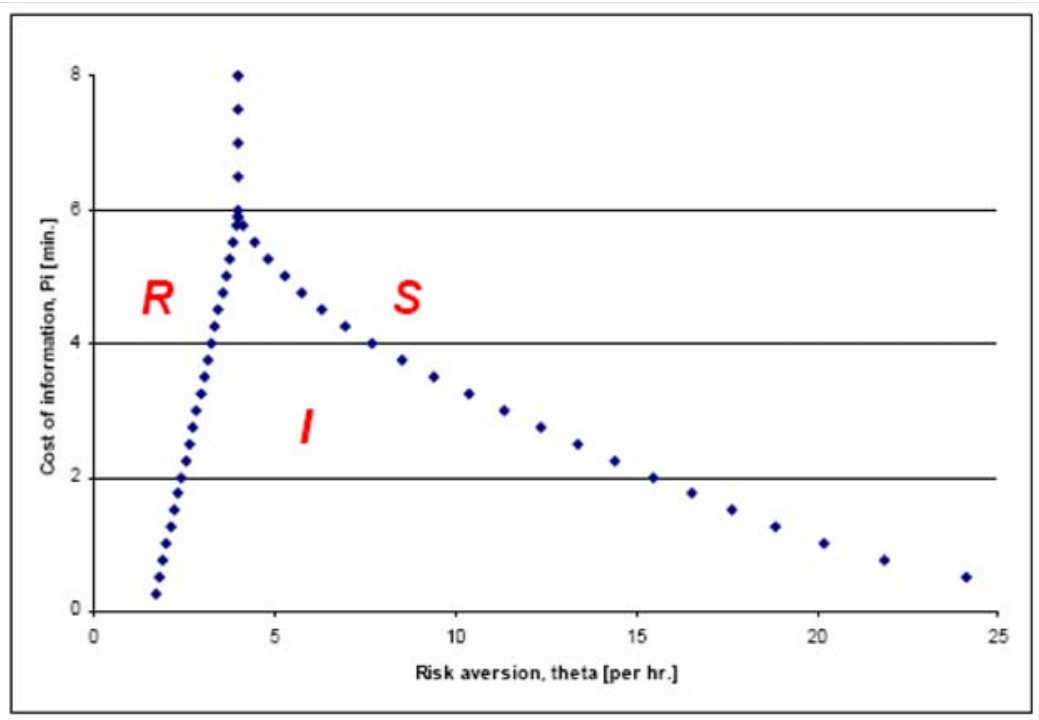

Figure 8: Strategy choice in Costly information equilibrium in numerical example 


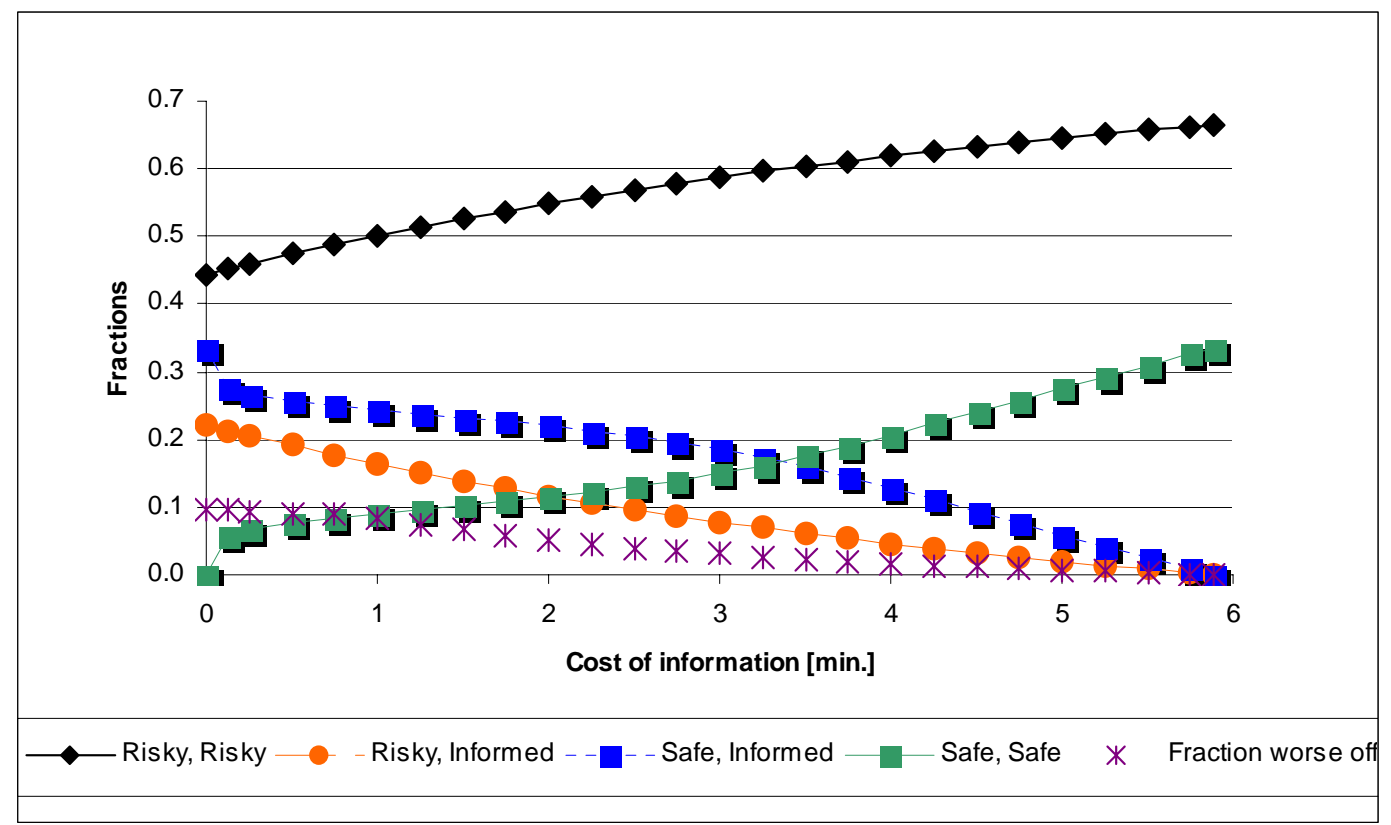

Figure 9: Route splits in numerical example

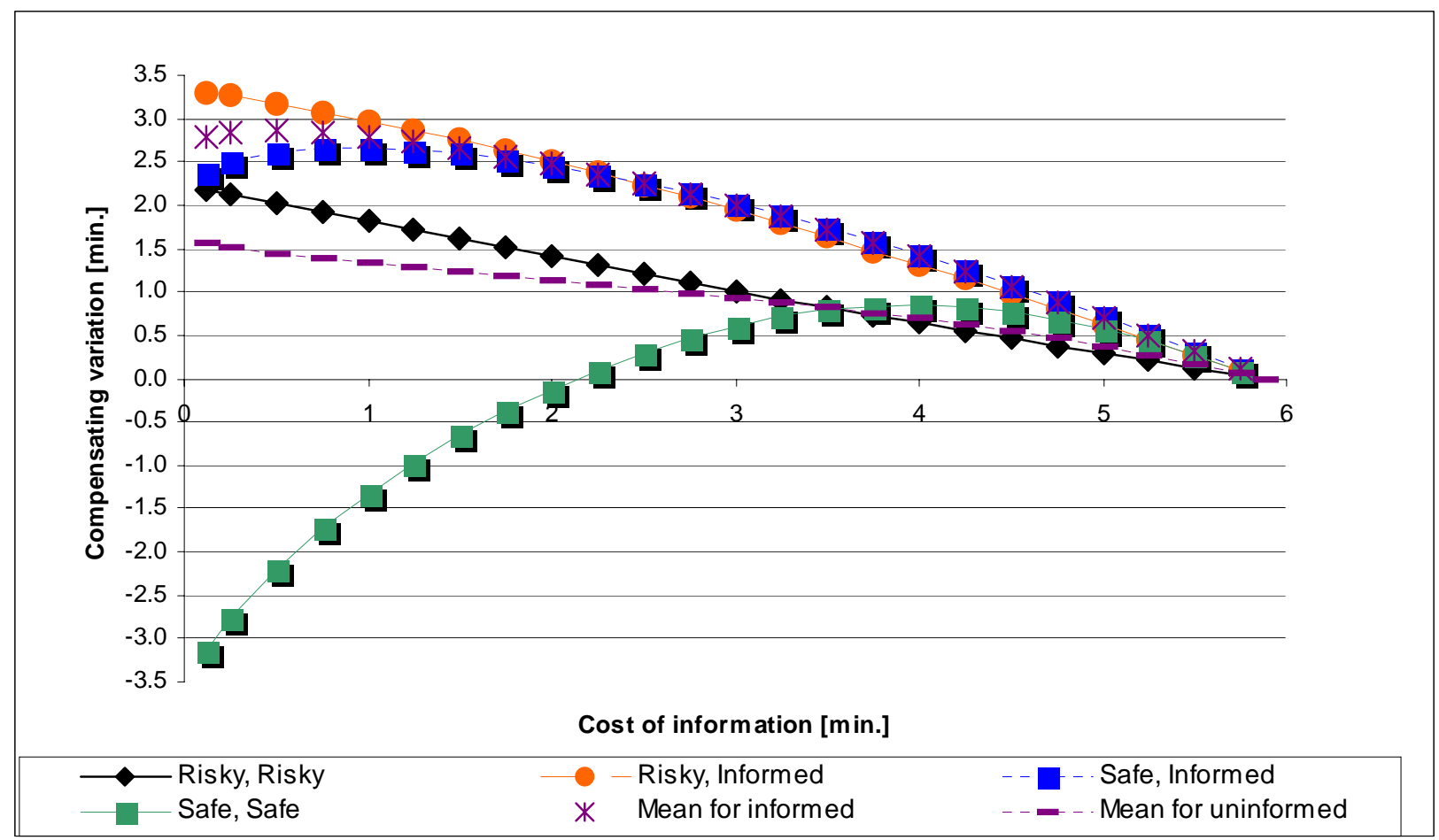

Figure 10: Compensating variation for Costly information in numerical example 


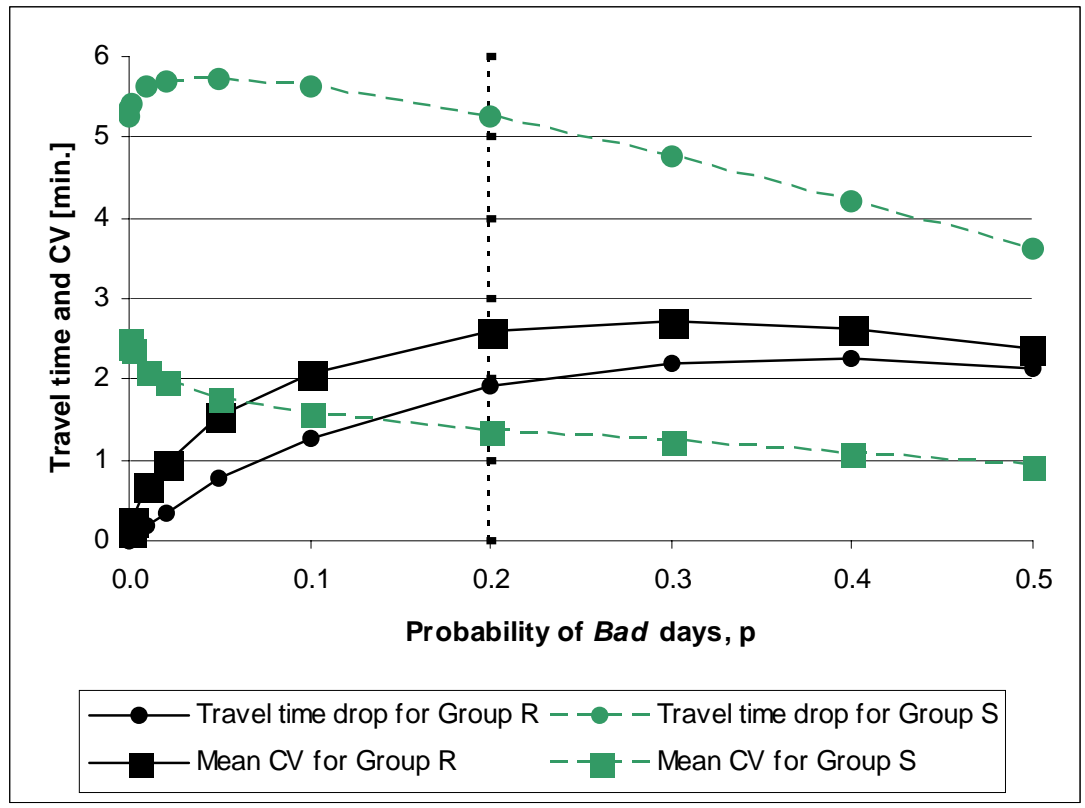

Figure 11: Varying probability of Bad days with Free information in numerical example

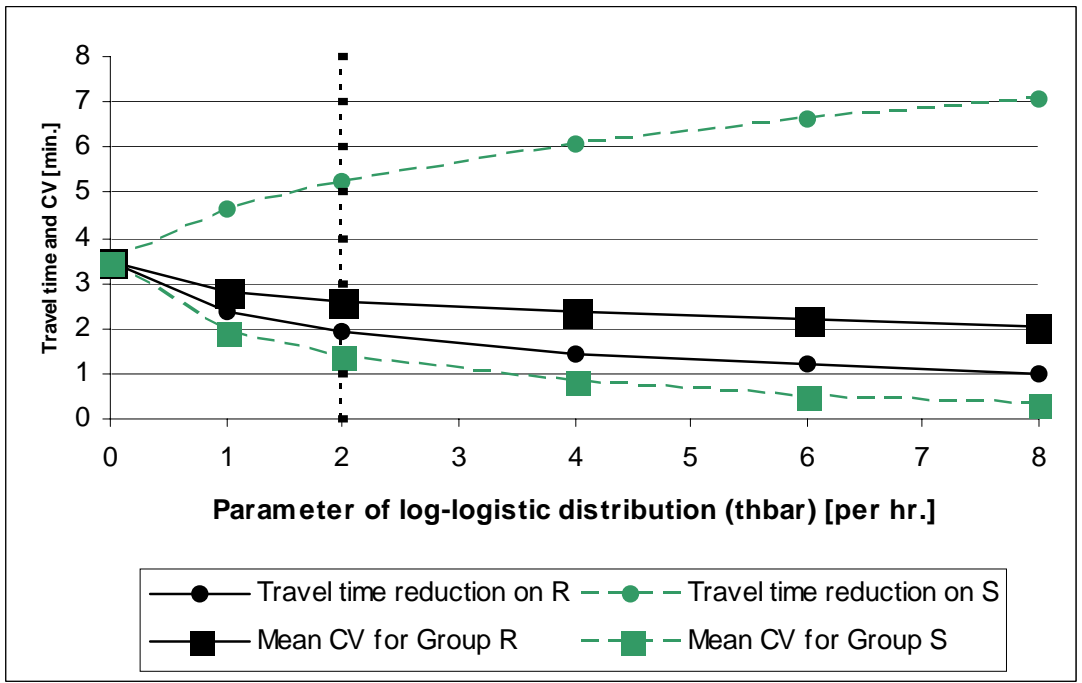

Figure 12: Varying parameter $\bar{\theta}$ with Free information in numerical example 